\title{
TITLE:
}

\section{Bus bunching along a corridor served by two lines}

$\operatorname{AUTHOR}(\mathrm{S})$ :

Schmocker, Jan-Dirk; Sun, Wenzhe; Fonzone, Achille; Liu, Ronghui

\section{CITATION:}

Schmocker, Jan-Dirk ...[et al]. Bus bunching along a corridor served by two lines. Transportation Research Part B: Methodological 2016, 93(7): 300-317

\section{ISSUE DATE:}

2016-11

URL:

http://hdl.handle.net/2433/252360

\section{RIGHT:}

(C) 2016. This manuscript version is made available under the CC-BY-NC-ND 4.0 license http://creativecommons.org/licenses/by-nc-nd/4.0/.; This is not the published version. Please cite only the published version.; この論文は出版社版でありません。引用の際には 出版社版をご確認ご利用ください。 
Schmöcker, J.-D., Sun, W., Liu, R. and Fonzone, A. (2016). Bus Bunching Along a Corridor Served by Two Lines. Transportation Research Part B, 93, 300-317; doi:10.1016/i.trb.2016.07.005

Also available from: http://authors.elsevier.com/a/1TVBDhVEAlH8n

\title{
BUS BUNCHING ALONG A CORRIDOR SERVED BY TWO LINES
}

\author{
Jan-Dirk SCHMÖCKER ${ }^{1}$, Wenzhe SUN ${ }^{1}$, Achille FONZONE² and Ronghui LIU ${ }^{3}$ \\ ${ }^{1}$ Department of Urban Management, Kyoto University, Japan; \\ email: \{schmoecker,wz.sun\} @trans.kuciv.kyoto-u.ac.jp \\ ${ }^{3}$ Transport Research Institute, Edinburgh Napier University, U.K.; \\ email: a.fonzone@napier.ac.uk \\ ${ }^{3}$ Institute for Transport Studies, University of Leeds, U.K.; \\ email: r.liu@its.leeds.ac.uk
}

\begin{abstract}
Headway fluctuations and "bus bunching" are well known phenomena on many bus routes where an initial delay to one service can disturb the whole schedule due to resulting differences in dwell times of subsequent buses at stops. This paper deals with the influence of a frequent but so far largely neglected characteristic of bus networks on bus bunching, that is the presence of overtaking and common lines. A set of discrete state equations is implemented to obtain the departure times of a group of buses following the occurrence of an exogenous delay to one bus at a bus stop. Two models are distinguished depending on whether overtaking at stops is possible or not. If two buses board simultaneously and overtaking is not possible, passengers will board the front bus. If overtaking is possible, passengers form equilibrium queues in order to minimise their waiting times. Conditions for equilibrium queues among passengers with different choice sets are formulated. With a case study we then illustrate that, if overtaking is not allowed, the presence of common lines worsens the service regularity along the corridor. Conversely, common lines have positive effects when overtaking is possible. We suggest hence that appropriate network design is important to reduce the negative effects of delay-prone lines on the overall network performance.
\end{abstract}




\section{Introduction}

The lack of bus service reliability is a major problem for bus passengers and service operators. A key feature of an unreliable service is the irregular arrivals of buses at stops. The effect of two successive services of a single line arriving at stops with shorter than designed headways is generally defined bus bunching. Bus bunching is undesirable for passengers because it reduces the predictability of bus arrival times and leads to on average increased waiting times at stops. This is particularly important sine studies have shown that passengers value their time waiting at bus stops more than they do to onboard travel time. For example, Hollander and Liu (2008) found that the value of service reliability to bus passengers is four times higher than that of mean travel time.

Bus bunching may be caused by the first service being delayed due to unforeseen traffic congestion en-route or unplanned high demand at previous stops. A further contributing factor is the differences in bus driver behaviour. If for any of these reasons a bus is delayed, the subsequent service then has fewer passengers to pick up at that stop and departs earlier than scheduled. At downstream stops the effect is emphasised as the (small) delay to the first vehicle and the (slight) early arrival of the second vehicle result in increasingly longer dwell times for the first bus and increasingly shorter dwell times for the second bus.

The bus bunching effect on a single line of service was first described in a seminal work by Newell and Potts (1964). They studied an idealised corridor with evenly spaced bus stops, identical travel times between stops, and constant passenger loads at bus stops. Given a small delay of the first bus at a stop, Newell and Potts provide an analytical formulation of the deviation of bus arrival time to schedule for all buses and at all subsequent stops. They show that adjacent buses alternate between behind and ahead of schedule, leading to bus bunching. The scale of the bunching effect and the stability of the bus system is affected not only by the size of the original delay to the first bus, but also by the ratio (referred to as the $k$ value later) between passenger arrival rate and loading rate. They show that if $1 / 2<k<1$, instability occurs. In practice, however, one would expect the passenger arrival rate to be much smaller than the loading rate, i.e. $0<k<1 / 2$. In this case, Newell and Potts show that the system can recover from the original perturbation and return to schedule. Potts and Tamli (1964) offered some empirical support, based on experimental investigations of bus bunching in Adelaide, Australia. They showed that the pairing of buses is in part due to the variations in passenger loading time. The analytical expression of Newell and Potts is in terms of the time a bus leaves a stop (see full description in Section 3). Chapman and Michel (1978) provided a different expression, in the form of the time between the departure of one bus from a stop and the arrival of the next. It is a more direct measure for bus pairing, and they used the method to identify the bus stop where bunching occurs. Since these earlier papers on bus bunching, much of the research has been to 
design and test means to control irregularities in bus operations so to reduce the bunching effect. In particular holding strategies for headway keeping and/or schedule-adherence have been analysed and shown to be successfully applied in literature. The holding objectives are different for low- and highfrequency services. For low-frequency systems, loosely defined as those that run at a headway of 10min or longer (Jolliffe and Hutchinson, 2001), holding strategies are implemented through building slacks in the schedule at key timing points and holding buses at these points to keep them to schedule (e.g. Osuna and Newell, 1971; Newell, 1974; Cats et al, 2012). For high-frequency systems, however, the holding strategies aim to maintain regularity in headways (e.g. Eberlein et al., 2001; Hickman, 2001). Due to the complexity of the problem, most of these early studies involve solving just one controlled timing point. Using a simulation approach, Hickman (2001) derived a set of static holding solutions, which do not respond to dynamical changes in the actual bus performances on the day. Eberlein et al (2001) proposed a model for dynamical bus holding which takes real-time information on bus headways into consideration and strives to minimise passenger waiting time. Liu and Sinha (2007) showed a clear correlation between headway regularity and passenger wait time delays.

Employing real-time bus positioning data, now widely available, Daganzo (2009) explored a more systematic approach to the dynamical holding problem. The method is able to consider holding at multiple timing points, therefore providing opportunity for returning to schedule for long bus route. In addition, the model takes into account random effects in bus travel time, bus dwell time and passenger demand, making it resemble more closely to real-life situations. Daganzo and Pilachowski (2011) proposed an adaptive bus control scheme based on a two-way bus-to-bus cooperation, where a bus adjusts its speed to both its front and rear headways. They show that the scheme yields significant improvements in bus headways and bus travel time. Pilachowski (2009) proposed to use GPS data to counteract directly the cause of the bunching by allowing the buses to cooperate with each other and to determine their speed based on relative position. Bartholdi and Eisenstein (2012) formalised the method as a self-coordinating strategy to equalise bus headway. Recently, Hernández et al. (2015) developed an optimal holding strategy, for a common-line corridor where two bus lines serve the same sub-set of stops. They showed that the holding strategy significantly reduced the overall waiting time of the passengers as well as reduced bus headway variation, compare to a no control scenario. Sun and Schmöcker (2016) analysed the effect of different passenger distributions on bus bunching. They show that an "ad hoc control strategy" whereby passengers are asked to board a latter bus could reduce the bunching effect. Their analysis is though also limited to buses of the same line, i.e. ignoring common lines.

Despite these recent developments, most of the existing studies present an oversimplified model of the bus bunching phenomenon, notably with a single line of service (with the exception of the recent work of Hernández et al. (2015)), with fixed service frequency, uniformly distributed (in time and 
space) passenger flows, and no bus overtaking. They neglect important aspects of real-life bus systems, such as passenger behaviour, en-route service perturbation, transport operator policies such as holding and overtaking, and complex network features such as common lines. Newell and Potts (1964), for instance, assume fixed frequency, constant dwell times, equal-distance stops and equaltravel time between stops, and that buses cannot overtake. In real-life situations, busy urban corridors are often served by multiple lines of bus services, with different frequencies and different sequence of stops. Traffic congestion causes uncertainty in bus run time, and buses overtake one another at bus stops. Passenger demand varies over time and between bus stops, which in turn lead to variation in bus dwell times. Boyd (1983) presented empirical evidence which demonstrated the impact of variability in bus journey time on bunching.

Another significant simplification in the existing studies is the assumption of random arrivals of passengers to bus stops, and the uniform passenger demand distribution over time and space. Bowman and Turnquist (1981) argue that passengers will, to some extent coordinate their arrivals to coincide with the scheduled service in an attempt to reduce their wait time, and that more reliable service would encourage such arrival behaviour. Using a passenger choice behaviour model, they demonstrate that passengers are more sensitive to schedule reliability than to service frequency. Nagatani (2001) shows a strong relationship between bus delay and the passenger number on bus, and proposed skipping a bus stop as a way of keeping to schedule. Liu and Sinha (2007) collected data on bus travel time, dwell time, and passenger boarding and alighting along a commuter bus route in the City of York, in England. They found that the passenger demand (both boarding and alighting) varies significantly by bus stops and over time. Sorratini et al (2008) show that the variability in passenger flow distribution has the most significant impact on bus reliability measures, as compared to that due to traffic congestion, overall passenger demand increases, or boarding rate.

Exploring the effect of non-uniform arrivals at stops on bus bunching, Fonzone et al (2015) developed a probabilistic reliability-based passenger arrival model in which passengers consider the scheduled as well as possible early or delayed bus departures in determining their arrival time and aim to minimize their expected wait time. They implement this probabilistic passenger arrival model with a standard bus propagation model (i.e. a single bus line, no holding or headway equalizing strategies), and show that a mismatch between the operators' perception of service demand and actual demand can lead to bus bunching, even without exogenous bus delay.

In this paper, we analyse bus bunching in a corridor with common lines. We focus on the effect of network layout, and more specifically that of the bus stop designs in the presence of common lines, on the absorption or propagation of an initial bus delay down the corridor. We consider the bus corridor 
served by two or more bus lines and investigate the spreading of bunching effect. The spreading can occur for two reasons in such a common line scenario. Firstly, if a large number of passengers interchange between lines at a certain point in the network and if a fully loaded vehicle arrives (for example after a special event or after re-opening of a line), this can lead to a sudden increase in demand for the line to which many passengers are transferring. Secondly, passengers' route choice may consider hyperpaths, i.e. sets of attractive lines, and if a specific line is delayed they choose an alternative option from their attractive set. Hence, irregular headways on one line can lead to large demand for the other lines.

Furthermore, we consider overtaking of buses at bus stops. We show that the Newell and Potts' model holds only when no bus overtaking is taken place and when no more than one bus can be at a stop at the same time. We formulate analytically the state equations for bus departure times on a corridor with common lines and allowing for bus overtaking at stops.

Section 2 of the paper sets out the basic model notations and illustrates the common line scenario to be considered. Section 3 presents the original Newell and Potts formulation of bus bunching and highlights its limitation with a numerical illustration. Section 4 describes the formulation and the state equations to the new bus propagation model in the presence of common lines, but where overtaking is not possible. Section 5 then considers the case where overtaking is allowed and a different and more complex passenger behaviour model considering queueing equilibria is developed. A number of evaluation measures are proposed in Section 6, and the performance of the new model are illustrated through case studies in Section 7. Finally, Section 8 draws conclusions of the study and discusses the implications on bus network design.

\section{Notation and basic assumptions}

\subsection{Notation}

The following notation will be used throughout the paper and explained in subsequent sections further as required. In parts we divert from those used in Newell and Potts (1964) in order to accommodate additional variables with intuitive notation as much as possible.

Let

$\begin{array}{ll}l & \text { bus line with } l=0,1, \ldots, \mathrm{L} ; \\ m(l) & \text { bus number of line } l \text { with } m(l)=0,1,2, \ldots, \mathrm{M}(l) \\ n(l) & \text { bus stop number of line } l \text { with } n(l)=0,1,2, \ldots, \mathrm{N}(l) \\ h_{l} & \text { headway of line } l\end{array}$


The above set of variables defines the basic service characteristics. We ignore alighting and capacity issues and focus on the effects of boarding demand on delay propagation. Given this restriction, in the following we introduce variables required to derive the bus trajectories. To simplify the notation we omit the line dependency of stops whenever possible, i.e. a bus $m(l)$ serves stops $n$ (not $n(l)$ ), even though clearly a bus serves only stops of its own line.

The following variables all have unit [sec]; and the times are measured from the departure of the first bus from terminal
$a_{m(l), n} \quad$ time at which bus $m$ of line $l$ arrives at stop $n$
$d_{m(l), n} \quad$ time at which bus $m$ of line $l$ leaves at stop $n$
$w_{m(l), n} \quad$ dwell time of bus $m$ of line $l$ at stop $n$
$v_{m(l), n} \quad$ travel time of bus $m$ of line $l$ between stops $n-1$ and $n$
$\rho_{m(l), n} \quad$ "exogenous" delay to bus $m$ of line $l$ at the $n$th stop
$\Delta_{\mathcal{L}, m(l), n} \quad$ passenger arrival period over which demand for bus $m(l)$ at stop $n$ accumulates assuming that passengers consider boarding line set $\mathcal{L}$ that includes line $l$ ( $l$ is used instead of $\mathcal{L}$ as first subscript when the passenger considers a single line only)
$\xi_{\mathcal{L}, m(l), n} \quad$ departure time of a bus of set $\mathcal{L}$ from stop $n$ immediate before bus $m(l)$

Further, we define:
$\mathcal{L}_{n}$
Set of lines considered for boarding by passengers at stop $n$
$\Omega, \Omega_{l} \quad$ Set of all $\mathcal{L}$ (set of sets of lines) and set of $\mathcal{L}$ that include line $l$
$b_{l} \quad$ passenger loading rate of buses of line $l[\mathrm{pas} / \mathrm{sec}]$
$k_{m(l), n} \quad$ Ratio between passenger arrival and loading for bus $m$ of line $l$ at stop $n$
$q_{\mathcal{L} n} \quad$ passenger arrival rate at stop $n$ for passengers with line set $\mathcal{L}$ [pas/sec]
$\tilde{q}_{n} \quad$ total passenger arrival rate at stop $n$ [pas/sec]
$\hat{y}_{m(l), n} \quad$ cumulative number of passengers that have boarded bus $m$ of line $l$ at stop $n$ when it departs

In addition we require following time depending variables:
$\hat{q}_{m(l), n}(t) \quad$ passenger arrival rate at stop $n$ intending to take line $l$ at time $t$ [pas/sec]
$y_{\mathcal{L}, n}(t) \quad$ cumulative number of passengers with choice set $\mathcal{L}$ that have arrived at stop $n$ at time $t$ since departure of a previous bus from $l \in \mathcal{L}$
$z_{\mathcal{L}, n}(t) \quad$ number of passengers with choice set $\mathcal{L}$ at stop $n$ at time $t$
$\hat{z}_{m(l), n}(t) \quad$ number of passengers who are waiting to board bus $m(l)$ at stop $n$ at time $t$ 


\subsection{Exogenous, initial delays triggering bunching}

We assume that bus travel time between stops are constant and equal so that $v_{m(l) n}$ simplifies to $v$. Introducing stochastic travel times on links where $v_{m(l) n}$ might be drawn from a (time-dependent) observed travel time distribution for a link does not constitute a modelling issue, nor does it alter the problem discussed in this paper. Instead, we assume that buses are subject to random delays at stops plus delays incurred by dwell times due to the bunching problem. The random exogenous part of the delays at stops is denoted by $\rho_{m(l) n}$. As we assume an uncontrolled bus service, any $\rho_{m(l) n} \neq 0$ triggers subsequent bunching effects. We note that the difference between assuming random link travel times and delays at stops is that in the latter passengers arriving at the stop during the delay period can board the bus whereas in the former they cannot.

\subsection{Illustration of common lines}

To illustrate the common line issue in connection with bus bunching, let's consider Figure 1. There are two lines originating from possibly two different terminals. After some stops the buses travel on a common corridor. Such a situation is frequently encountered where buses depart from suburbs and then travel on an arterial street in the city centre. To illustrate the network design issue, two types of stops are distinguished on the common corridor. At Stops 1 and 2 the buses board passengers at nearby but different stop locations so that passengers have to decide for a specific bus at the point of arrival. Such bus stop designs are common in practice. For example, the stop for the blue line is located before a road-crossing whereas the stop for the red line is located after the crossing. Another type of bus stops, as for the case of Stop 3 in Fig. 1, there is only one stopping point for both lines so that passengers form a single queue and board whichever bus arrives first and such bus stops can also be frequently found in practice. We assume that the buses travel together for a certain section before they might split again. This means that only a proportion of passengers, i.e. those travelling up to stop $n$ will be able to take advantage of the same stop being served by both lines. 


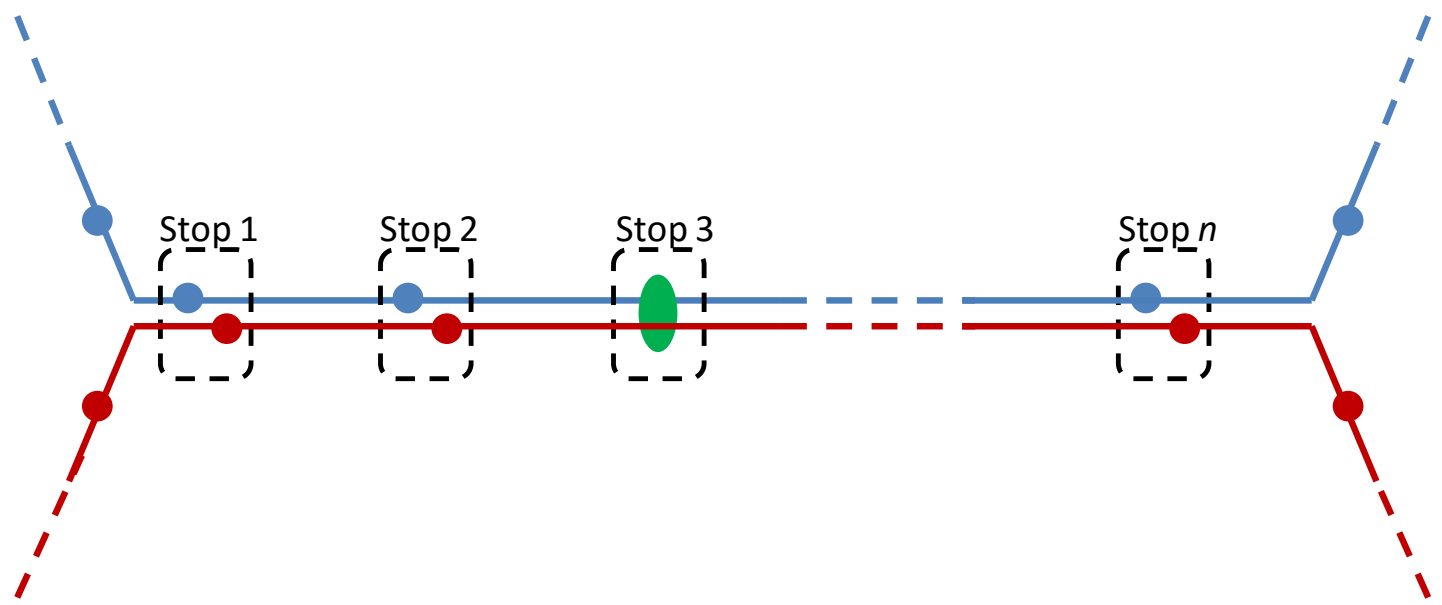

Figure 1. Illustration of two bus lines with some common line stops

It is reasonable to assume that the passenger arrival pattern is independent of the bus stop layout. To describe the effect of common line stops we distinguish the set of lines $\mathcal{L}$ different passenger groups might be considering in their choice set. Let $\boldsymbol{q}_{\mathcal{L} n}$ denote the set of passenger arrival rates at stop $n$ for passengers considering to board a line among set $\mathcal{L}$. For $n$ lines serving the stop we have $2^{n}-1$ choice sets. In Figure 1 we hence need to distinguish three passenger group arrival rates so that $\boldsymbol{q}_{\mathcal{L n}}$ becomes a vector of $\left(q_{\{\text {red }\} n}, q_{\{b l u e\} n}, q_{\{r e d, b l u e\} n}\right)$.

Let $\tilde{q}_{n}$ further denote the total passenger arrival rate at stop $n$. We then obtain for non-common line stops, such as Stops 1 and 2 in Figure 1, that passengers have to decide for a particular line upon arrival at the stop and therefore obtain $\tilde{q}_{n}=q_{\{\text {red }\} n}+q_{\{\text {blue }\} n}$, and $q_{\{\text {red,blue }\} n}=0$. In case no schedule is available and all lines serve the same downstream stops it might be assumed that passengers at non-common line stops will split in inverse proportion to the line headway $h_{l}$ so that the loads on all buses are evenly distributed, i.e.

$$
q_{\{l\} n}=\tilde{q}_{n} \frac{1 / h_{l}}{\sum_{l^{\prime} \in L^{1}} 1 / h_{l^{\prime}}} .
$$

In this paper we follow this assumption though we note that other distributions might also be reasonably assumed. For example, in an uncongested situation, it is not unreasonable to assume that all passengers might always go to the stop served by the more frequent service.

\subsection{Boarding demand for buses}

The total boarding demand for a single bus $m$ of line $l$ at stop $n$ is obtained by (2)

$$
\hat{y}_{m(l), n}=\sum_{\mathcal{L} \in \Omega_{l}} \int_{t^{\prime}}^{t^{\prime}+\Delta_{\mathcal{L}, m(l) n}} q_{\mathcal{L} n}(t) d t
$$


where $t^{\prime}$ denotes the time from where passengers at the stop could not (or do not) board the previous bus at the same stop anymore. Ignoring capacity constraints and assuming uncontrolled boarding, i.e. that passengers who arrive during the dwell time can still board the front bus one would hence generally expect that $t^{\prime}$ equals the departure time of the previous bus from the stop. In contrast to Fonzone (2015) we consider frequent services for which time-independent arrival rates can be assumed so that (2) simplifies to

$$
\hat{y}_{m(l), n}=\sum_{\mathcal{L} \in \Omega_{l n}} q_{\mathcal{L} n} \Delta_{\mathcal{L}, m(l) n}
$$

We note that in case of a common lines stop the period $\Delta_{\mathcal{L}, m(l) n}$ reduces for each bus: Consider that two lines serve the stop, for both lines $\Delta_{\mathcal{L}, m(l) n}$ reduces on average from $h_{1}$ and $h_{2}$ respectively to the combined line frequency of $\frac{1}{h_{1}}+\frac{1}{h_{2}}=\frac{h_{1} h_{2}}{h_{1}+h_{2}}$.

LEMMA 1: The total amount of passengers boarding over a time period $T$ remains the same regardless whether the bus stop is designed as a common line stop or not:

\section{Proof:}

In case the stop is not a common lines stop $q_{\{1,2\} n}=0$ and we expect $q_{\{l\} n} h_{l}$ passengers per vehicle so that the total number of passengers boarding over a period T is: $\left(q_{\{1\} n} h_{1}\right)\left(\frac{T}{h_{1}}\right)+$ $\left(q_{\{2\} n} h_{2}\right)\left(\frac{T}{h_{2}}\right)=\left(q_{\{1\} n}+q_{\{2\} n}\right) T=q_{n} T$.

In case the stop is a common lines stop we expect instead on average for both lines $l$ a passenger load of $\left(q_{\{l\} n} h_{l}\right)+\frac{h_{1} h_{2}}{h_{1}+h_{2}} q_{\{1,2\} n}$ and hence the total number of passengers boarding over a period $\mathrm{T} \quad$ is $\quad$ also: $\quad\left(q_{\{1\} n} h_{1}\right)\left(\frac{T}{h_{1}}\right)+\left(q_{\{2\} n} h_{2}\right)\left(\frac{T}{h_{2}}\right)+\left(\frac{h_{1} h_{2}}{h_{1}+h_{2}} q_{\{1,2\} n}\right)\left(\frac{T}{h_{1}}+\frac{T}{h_{2}}\right)=\left(q_{\{1\} n}+q_{\{2\} n}+\right.$ $\left.q_{\{1,2\} n}\right) T=q_{n} T$

This completes the proof of Lemma 1.

The period, $\Delta_{\mathcal{L}, m(l) n}$, over which passengers for set $\mathcal{L}$ accumulate will depend on the departure of a previous bus from the set $\mathcal{L}$ as well as the arrival and (expected) departure of the next bus from this set. In case of a bunched service various definitions are possible, depending on bus stop layout, operational policy as well as passenger behaviour. In particular, one might make different assumptions on the behaviour of passengers arriving while two buses are at the same time at the stop as will be discussed later in this paper. 


\subsection{Demand ratio $k_{l n}$}

The boarding time per passenger is primarily depending on doors and ticketing system. Sun et al (2014) report that the loading time per passenger further depends on the interaction between boarding and alighting passengers. In the following we omit this issue and instead make the simplifying assumption that all buses are identical, i.e. have the same boarding rate per passenger, so that we can assume a fixed $b_{m(l)}$ and omit the subscript $m(l)$. In line with Newell and Potts at non-common line stops (or at common line stops with $q_{\mathcal{L} n}=0$ for all line sets $\mathcal{L}$ that include several lines) we can hence derive a bus independent "static" and dimensionless demand ratio $k_{l n}$ that can be used to obtain dwell time of buses:

$$
k_{l n}=\frac{q_{l n}}{b}
$$

Clearly to avoid queues at bus stops building up over the analysis period we require

$$
0 \leq k_{l n}<1
$$

In case of common line stops with passengers utilising different line sets $q_{\{\mathcal{L}\} n}$ that include the same line $l$, the use of a bus independent demand ratio ignores the fact that different arrival periods $\Delta_{\mathcal{L}, m(l) n}$ need to be considered to obtain the dwell time at stops. Instead considering the dynamics of line choice for passengers depending on their choice set $\mathcal{L}$ is required to obtain boarding demand for buses and with it dwell time.

\section{Limitations of the Newell and Potts (1964) approach}

We start by reviewing the Newell and Potts (1964) model as one of the most cited papers and seminal works on the bunching problem for the single line problem. Their approach is based on the assumption that Loading time $=$ Arrival period $x$ Arrival rate ${ }^{l}$. With the above-introduced notation utilising the ratio $k_{l n}$ they derive:

$$
w_{m(l), n}=\Delta_{l, m(l), n} k_{l n}
$$

Where, since common lines are not considered, arrival periods $\Delta$ are defined as:

$$
\Delta_{l, m(l), n}=d_{m(l), n}-d_{m-1(l), n}
$$

\footnotetext{
${ }^{1}$ See also Figure 2 in Newell and Potts (1964). Readers of both papers might note that Newell and Potts call "arrival time" what we refer here as "arrival period".
} 
The implicit assumption is that passengers keep boarding a bus until it departs. In other words, the issue that passengers might prefer to board a bus that arrived later (but might already be in sight or even already at the bus stop) is not considered. This assumption is however difficult to defend if overtaking is allowed and a bus arriving later might in fact leave the bus stop earlier. This is the basis for the model presented in Section 5. Further note that:

$$
w_{m(l), n}=d_{m(l), n}-d_{m(l), n-1}-v
$$

With this (6) can now be used to solve the cyclic problem between obtaining $\Delta_{l, m(l) n}$ and $d_{m(l) n}$. That is, if (7) and (8) are inserted into (6) one can derive:

$$
d_{m(l), n}=\frac{v+d_{m(l), n-1}-d_{m-1(l), n} k_{l n}}{1-k_{l n}}
$$

With this formulation Newell and Potts then derive their elegant, analytically tractable formulation of $d_{m(l) n}$ given that $k_{l n}$ reduces to a constant $k$ and that the first bus $m=1$ on line $l$ is delayed at stop $n=1$ by $\rho_{1(l) 1}$ as following:

$$
d_{m(l), n}=(m+n k) h_{l}+n v+\rho_{1(l) 1} \frac{(n+m-1) !}{(n-1) !(m-1) !}\left[\frac{k}{k-1}\right]^{m-1}\left[\frac{1}{1-k}\right]^{n-1}
$$

Based on this, it follows that if $k \geq 0.5$ the bunching effect increases, whereas for $k<0.5$ the system can recover from perturbations. In addition to the assumptions common with our subsequent approach, a number of restrictions need to be noted though on which this expression of bus departure times is built: a) the formulation (9) and the resulting equation (10) do not consider issues such as overtaking; b) if buses are bunched and more than one bus is at the platform, arrival period and loading time estimation is not true; c) the result given in (10) does not hold if several delays occur. Points a) and b) lead to the problems that are illustrated in the following figure even assuming only mild bunching conditions. Applying (10) for a headway of $h_{l}=5 \mathrm{~min}$, a travel time between stops of $v=2 \mathrm{~min}$, an initial delay of $\rho_{1(l) 1}=1 \mathrm{~min}$ and a constant $k$ value of 0.2 , Fig. 2 shows that it takes just two bus stops before bus 2 catches bus 1. Afterwards, according to the Newell and Potts model, the trajectories of bus 2 reverse in time, which is clearly not realistic. 


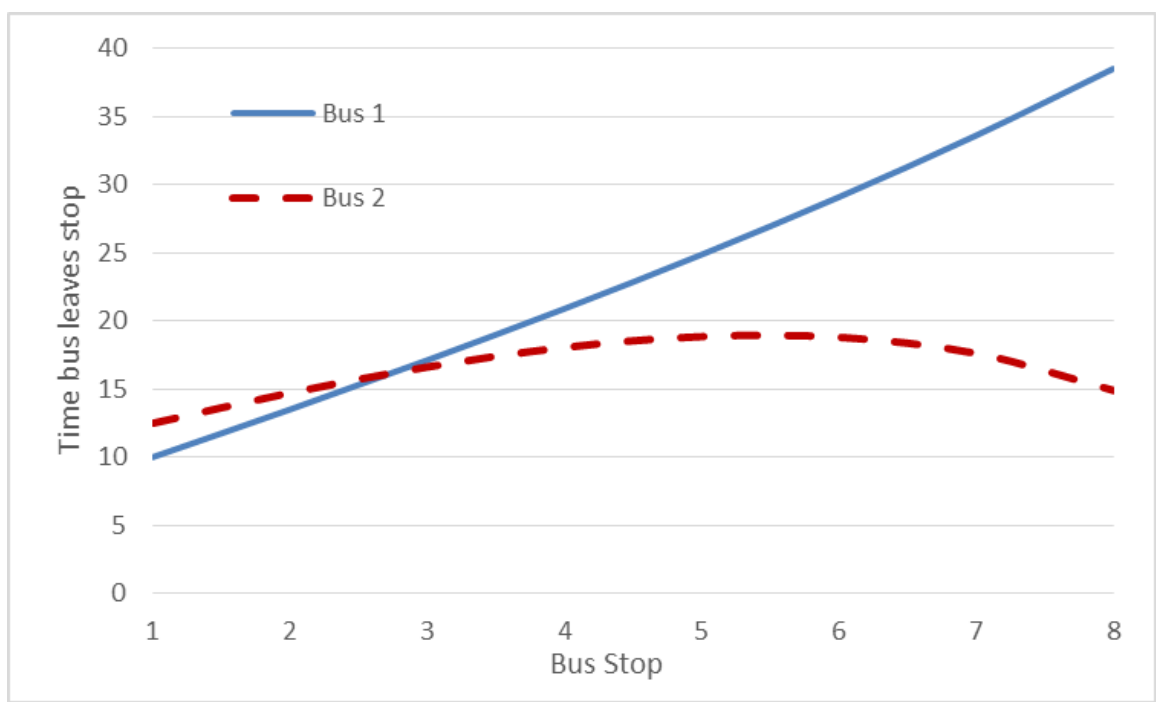

Figure 2. Trajectories of two buses according to Newell and Potts (1964)

Giving the assumptions discussed in Section 2.3 and our objective to consider common lines, we modify (7) into:

$$
\Delta_{\mathcal{L}, m(l), n}=d_{m(l), n}-\xi_{\mathcal{L}, m(l), n}
$$

where we introduce $\xi_{\mathcal{L}, m(l), n}$ as shorthand for the previous departure from stop $n$ from a bus of set $\mathcal{L}$ prior to bus $m(l)$ arrives at the stop.

$$
\xi_{\mathcal{L}, m(l), n} \equiv \max _{m^{\prime}\left(l^{\prime} \in \mathcal{L}\right)}\left\{d_{m^{\prime}\left(l^{\prime}\right), n} \mid d_{m^{\prime}\left(l^{\prime}\right), n} \leq a_{m(l) n}\right\} \forall m\left(l^{\prime}\right), l^{\prime} \in \mathcal{L}_{n}
$$

The passenger arrival period for a bus $m$ of line $l$ is hence shortened as the time period that has passed since the departure of a previous bus from any line in $\mathcal{L}$ and the departure of the current bus. We observe though that an equivalent formulation to (10) cannot be derived if we use (11) instead of (7).

In summary, we suggest the contribution of the Newell and Potts (1964) model is to illustrate the theoretical tendency of $k$ to increase bunching. In particular Newell and Potts show that $k=0.5$ is a threshold. For smaller $k$ self-recovery can be expected, but for larger $k$ it can not. However, the model is not suitable to predict arrival times of a series of buses at stops. Our objective is to address these points and to take into account an arrival period definition that considers common lines. In the following we therefore propose an alternative formulation that considers these points. We avoid a simulation approach, but the limitation of our study is that in contrast to Newell and Potts we are not able to derive a formulation equivalent to (10). Instead we develop a recursive analytical formulation of $d_{m(l) n}$.

Proposition 1: Increasing the sequence of common line stops reduces the maximum delay of the first delayed bus for a constant $k_{l n}$. 
Proof: See the Appendix.

Due to the dynamic interactive behaviours between passengers' line choices at the common line stops, a more general relationship between common line stop and passenger delays than in Proposition 1 cannot be formulated analytically. Instead, we examine in Section 6 numerically the effect of common line stops and show a general trend in delay reduction when common lines are considered.

\section{Bus propagation model in case of no overtaking (assuming only one bus at a time can load passengers)}

Generally, based on (4) and (6), dwell times at a stop under consideration of common lines can be obtained by:

$$
w_{m(l), n}=\frac{1}{b} \sum_{\mathcal{L} \in \Omega_{l n}} \int_{t^{\prime}}^{t^{\prime}+\Delta_{\mathcal{L}, m(l) n}} q_{\mathcal{L} n}(t) d t
$$

The treatment of common lines is considered in the definition of the integration interval of (13). With our previously introduced definition of $\Delta_{\mathcal{L}, m(l) n}$ this can also be expressed as:

$$
w_{m(l), n}=\frac{1}{b} \sum_{\mathcal{L} \in \Omega_{l n}} \int_{\xi_{\mathcal{L}, m(l), n}}^{d_{m(l), n}} q_{\mathcal{L} n}(t) d t
$$

As noted before, the implicit assumption of (14) is that if two buses are at the same stop, passengers board the front bus until this departs. This is a typical situation for stops where bus bays are only large enough for one bus to board passengers. In that case passengers will know that the first bus will also arrive earlier at subsequent stops and therefore have no motivation to board the second bus (considering travel time only). With (14) and utilising our assumption of uniform passenger arrivals we then derive (15) where dwell time appears on both the right and left hand side of the equation but which can be easily solved for $w_{m(l), n}$.

$$
\begin{aligned}
& w_{m(l), n}=\frac{1}{b} \sum_{\mathcal{L} \in \Omega_{l n}} \int_{\xi_{\mathcal{L}, m(l), n}}^{d_{m(l), n}} q_{\mathcal{L} n}(t) d t=\frac{1}{b} \sum_{\mathcal{L} \in \Omega_{l n}} \int_{\xi_{\mathcal{L}, m(l), n}}^{a_{m(l), n}+w_{m(l), n}} q_{\mathcal{L} n} d t= \\
& \frac{1}{b} \sum_{\mathcal{L} \in \Omega_{l n}} q_{\mathcal{L} n}\left(a_{m(l), n}+w_{m(l), n}-\xi_{\mathcal{L}, m(l), n}\right)
\end{aligned}
$$

Note that (15) does not hold if the assumption of passenger uniform arrival is not valid or if boarding rates are not constant, i.e. if buses board passengers slower when the bus is crowded. In this case, one will have to revert to a "time-step based simulation" to solve the cyclic relationship between the arrival rate and the departure time as in Fonzone et al (2015). 
The formulation of (15) is then key to obtain our dynamic state equations that describe the propagation of buses through the network without consideration of overtaking at stops. This is shown below in Algorithm 1.

Algorithm 1: Bus trajectories without overtaking

Initialisation

Set $a_{m(l), 1} \forall m(l), l \quad$ the arrival times of all buses of line $l$ at stop 1

Set $\Delta_{\mathcal{L}, 1(l), n} \forall n(l), l \quad$ the waiting times for the first bus at all stops on line $l$

For each stop $n$ in increasing order

Sort buses according to their arrival times at the stop

For each bus $m \in \mathcal{L}$ in order of increasing arrival times, obtain $\xi_{\mathcal{L}, m(l), n}$ with (12). Then

$$
\begin{aligned}
& w_{m(l) n}=\left\{\begin{array}{cc}
\frac{1}{b} \sum_{\mathcal{L} \in \Omega_{l n}} q_{\mathcal{L} n} \Delta_{\mathcal{L}, 1(l), n} & m=1 \\
\frac{1}{b} \sum_{\mathcal{L} \in \Omega_{l n}} q_{\mathcal{L} n}\left(a_{m(l), n}+w_{m(l), n}-\xi_{\mathcal{L}, m(l), n}\right) & m>1
\end{array}\right. \\
& d_{m(l) n}=a_{m(l), n}+\max \left\{\xi_{\mathcal{L}, m(l), n}+\varepsilon, w_{m(l), n}\right\}+\rho_{m(l), n} \\
& a_{m(l), n+1}=d_{m(l), n}+v_{m(l), n}
\end{aligned}
$$

In the initialisation, the arrivals of the buses at the first stops are predetermined. One might interpret them as the time the bus leaves the terminal. Furthermore, we assume that the waiting times for the first bus of each line are known and given. Thus, assuming that the service is initially undisturbed and hence $\Delta_{\mathcal{L}, 1(l), n}=h_{l}$ for all lines and stops, we can obtain the same dwell time for the first bus at all stops in the corridor, using the first part of eq. (16). The second part of (16) gives the state equation for calculating the dwell times of subsequent buses.

Dealing with stops in increasing sequence ensures that the departure times of all buses at previous stops have been obtained. In line with Figure 1 we assume that all buses serve all stops. This is mainly in order to allow simplification of notation as otherwise one would need $n(l)$. If the assumption does not hold, one can set the arrival rate (and hence dwell time) for that stop to zero, so that this is not a restricting assumption.

Eq. (17) includes a max operator in order to ensure that buses depart in the same order as they arrived at the bus stop, i.e. no overtaking at bus stops is allowed. We further add a small time $\varepsilon$ (a few seconds) to the departure time of bus $m$ in case it could depart earlier or at the same time as the bus 
loading in front. This is in order to prevent exactly equal arrival times of two buses (and for better illustration of the bus trajectories shown later). As noted, delays are assumed to occur at stops and are hence added to the departure time in (17). If instead/additionally random link travel times are assumed these can be added to (18). Note that in that case random overtaking between stops might occur, this remains in line with model assumptions presented in this section: As long as passengers cannot expect the latter bus to overtake a bus queuing at the stop in front, passengers will prefer the front one (ignoring capacity problems).

\section{Bus propagation model with overtaking at stops}

\subsection{Larger bus bays and resulting passenger grouping}

Let us now consider the case of bus bays being large enough for two buses to board passengers at the same time and/or generally overtaking between buses being possible. In that case we consider that, if possible, passengers at the stop form equally long queues for the two buses that are boarding passengers at the same time. This appears reasonable as it will mean equal waiting times for all passengers and minimises the waiting time for the last passenger at the stop. The equilibrium assumption on bus bay layout and passenger behaviour also means that the assumption of no overtaking is not reasonable anymore as the second bus at the stop might board fewer passengers and therefore can leave the stop earlier. Therefore in (16) the max operator can be omitted so that obtaining bus departure times simplifies to

$$
d_{m(l) n}=a_{m(l), n}+w_{m(l), n}+\rho_{m(l), n}
$$

For obtaining the dwell time of bus $m(l)$ now several cases need to be distinguished though. To simplify the notations, in this section we omit subscript $n$ as all notation will always refer to the same stop. To begin with, let us assume two successive buses arrive at stop $n$, and if it is a common-line stop, they do not necessarily belong to the same line. Hence the arrival time of these two services can be denoted by $a_{m(l)}$ and $a_{m^{\prime}\left(l^{\prime}\right)}$.

The passengers waiting to board at the stop can now be split into four groups according to their line choice set $\Omega$ which will determine their queueing behaviour: Those who are not interested in boarding any of the two lines $\left(\Omega_{\neg l, \neg l^{\prime}}\right)$, those with $\mathcal{L}$ that includes exactly one of the two lines $\left(\Omega_{l, \neg l^{\prime}}\right.$ and $\left.\Omega_{\neg l, l^{\prime}}\right)$ and the common line passengers whose line sets concludes both $l$ and $l^{\prime}\left(\Omega_{l, l^{\prime}}\right)$.

Whereas the group with choice set $\Omega_{\neg l, \neg l^{\prime}}$ will not queue for any bus, the other three passenger groups will now split into two queues for the two buses at the stop. Passengers with $\Omega_{l, \neg l^{\prime}}$ and $\Omega_{\neg l, l^{\prime}}$ will 
form a queue for the single bus they wish to board. Passengers $\Omega_{l, l^{\prime}}$ though have the choice to join any one of the two queues. It is reasonable to assume that these passengers will choose to join the shorter queue in order to speed up their departure from the stop.

\subsection{Necessary condition for two buses loading at the same time}

In order for the case of two buses boarding passengers simultaneously to occur, the boarding process of the bus that arrived first must not have finished at the time when the second bus arrives. Assume that buses of line $l$ and $l^{\prime}$ board simultaneously and that the bus of line $l$ arrived first. Then, at $a_{m^{\prime}\left(l^{\prime}\right)}$ following condition applies:

$$
y_{\mathcal{L} \in \Omega_{l, \neg l^{\prime}}}\left(a_{m^{\prime}\left(l^{\prime}\right)}\right)+y_{\mathcal{L} \in \Omega_{l, l^{\prime}}}\left(a_{m^{\prime}\left(l^{\prime}\right)}\right)>b\left(a_{m^{\prime}\left(l^{\prime}\right)}-a_{m(l)}\right)
$$

(20) states that the boarding demand for line $l$ must exceed the total number of passengers arrived during the period between $a_{m(l)}$ and $a_{m^{\prime}\left(l^{\prime}\right)}$. Where we remind that $y_{\mathcal{L}}(t)$ denotes the accumulated demand for passengers of the respective groups since a previous departure of a bus from choice sets $\mathcal{L}$. These can be obtained generally from (21) where $\Omega_{x}$ can be replaced by $\Omega_{l, \neg l^{\prime}}$ or $\Omega_{l, l^{\prime}}$ respectively.

$$
y_{\mathcal{L} \in \Omega_{x}}\left(a_{m^{\prime}\left(l^{\prime}\right)}\right)=\sum_{\mathcal{L} \in \Omega_{x}} \int_{\xi_{\mathcal{L}, m(l), n}}^{a_{m^{\prime}\left(l^{\prime}\right)}} q_{\mathcal{L}}(t) d t=\sum_{\mathcal{L} \in \Omega_{x}} q_{\mathcal{L}}\left(a_{m^{\prime}\left(l^{\prime}\right)}-\xi_{\mathcal{L}, m(l), n}\right)
$$

\subsection{Queues at the arrival of the second bus}

In order to define the length of the two queues we firstly define the number of passengers remaining from each group at the stop at time $a_{m^{\prime}\left(l^{\prime}\right)}$ as $z_{\mathcal{L} \in \Omega_{l, \neg l^{\prime}}}\left(a_{m^{\prime}\left(l^{\prime}\right)}\right)$ :

$$
\begin{aligned}
& z_{\mathcal{L} \in \Omega_{l, \neg l^{\prime}}}\left(a_{m^{\prime}\left(l^{\prime}\right)}\right)=\left[y_{\mathcal{L} \in \Omega_{l, \neg l^{\prime}}}\left(a_{m^{\prime}\left(l^{\prime}\right)}\right)+y_{\mathcal{L} \in \Omega_{l, l^{\prime}}}\left(a_{m^{\prime}\left(l^{\prime}\right)}\right)-b\left(a_{m(l)}-\right.\right. \\
& \left.\left.a_{m^{\prime}\left(l^{\prime}\right)}\right)\right] \frac{y_{\mathcal{L} \in \Omega_{l, \neg l^{\prime}}}\left(a_{m^{\prime}\left(l^{\prime}\right)}\right)}{y_{\mathcal{L} \in \Omega_{l, \neg l^{\prime}}}\left(a_{m^{\prime}\left(l^{\prime}\right)}\right)+y_{\mathcal{L} \in \Omega_{l, l^{\prime}}}\left(a_{m^{\prime}\left(l^{\prime}\right)}\right)}
\end{aligned}
$$

The square bracket denotes the total remaining passengers with an interest in boarding line $l$. This

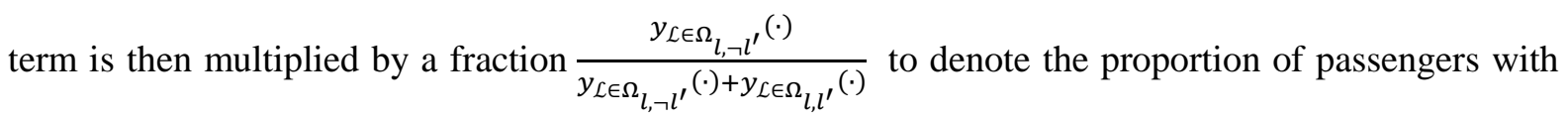
choice set $\Omega_{l, \neg l^{\prime}}$ that have boarded. The underlying assumption is that there is no ordering in the queue between passengers with different choice sets. To obtain $z_{\mathcal{L} \in \Omega_{l, l^{\prime}}}\left(a_{m^{\prime}\left(l^{\prime}\right)}\right)$ one hence has to replace the fraction by $\frac{y_{\mathcal{L} \in \Omega_{l, l^{\prime}}}(\cdot)}{y_{\mathcal{L} \in \Omega_{l, \neg l^{\prime}}}(\cdot)+y_{\mathcal{L} \in \Omega_{l, l^{\prime}}}(\cdot)}$. Further note that obviously (23) holds since bus $m^{\prime}\left(l^{\prime}\right)$ has just been arriving. 


$$
z_{\mathcal{L} \in \Omega_{\neg l, l^{\prime}}}\left(a_{m^{\prime}\left(l^{\prime}\right)}\right)=y_{\mathcal{L} \in \Omega_{\neg l, l^{\prime}}}\left(a_{m^{\prime}\left(l^{\prime}\right)}\right)
$$

As noted, we assume common line passengers are supposed to join the shorter queue. This means that, if the proportion of common line passengers is large, the common line passengers will distribute themselves in such a way as to create queues of equal length. If, however, the proportion of noncommon line passengers is too large equal queues might not be reached. This rule can be expressed simply with (24):

$$
\hat{z}_{m(l)}(\cdot)=\min \left(\max \left(\sum_{\mathcal{L} \in \Omega_{l, \neg l^{\prime}}} z_{\mathcal{L}}(\cdot), \sum_{\mathcal{L} \in \Omega} z_{\mathcal{L}}(\cdot) / 2\right), \sum_{\mathcal{L} \in \Omega_{l}} z_{\mathcal{L}}(\cdot)\right)
$$

where $(\cdot)$ will be time $a_{m^{\prime}\left(l^{\prime}\right)}$ in our case. The three values in the function above are illustrating three different queue situations: When it is possible for the two queues to equalize, we obtain $\sum_{\mathcal{L} \in \Omega} z_{\mathcal{L}}(\cdot) / 2$ as queue length for the bus of line $l$, if the queue for line $l$ is longer than for line $l^{\prime}$ we obtain $\sum_{\mathcal{L} \in \Omega_{l, l^{\prime}}} z_{\mathcal{L}}(\cdot)$ and for the opposite condition we obtain $\sum_{\mathcal{L} \in \Omega_{l}} z_{\mathcal{L}}(\cdot)$. The max operator covers the case that the equilibrium situation is not possible because there is too much demand restricted to boarding line $l$. The min operator then controls for the lower bound of passengers that can board line $l$ which is either the equilibrium case or the case that all passengers with line $l$ included in their choice set board line $l$.

To illustrate this, assume that there are two bus lines serving the stop and that bus $m$ belongs to line 1, then (24) becomes

$$
\hat{z}_{m(1)}(\cdot)=\min \left(\max \left(z_{\{1\} n},\left(z_{\{1\} n}+z_{\{2\} n}+z_{\{1,2\} n}\right) / 2\right), z_{\{1\} n}+z_{\{1,2\} n}\right)
$$

Assume further that a bus of line 1 has arrived earlier and that at time $t$ a bus of line 2 arrives. Let $z_{\{1,2\}}(t)=8, z_{\{1\}}(t)=10, z_{\{2\}}(t)=6$ so that 18 passengers have been queueing for line 1 at time $t$. It follows that $\min \left(8, \frac{18-6}{2}\right)=6$ passengers will swap queue to the second one, leaving $10+$ $\max \left(0, \frac{8+(6-10)}{2}\right)=10+2=12$ waiting to board the first bus, and $6+\min \left(8, \frac{18-6}{2}\right)=6+6=12$ queuing for the second bus. In this equilibrium is reached. If, however, $z_{\{2\}}(t)=0$, i.e. all waiting passengers consider taking line $1, \min \left(8, \frac{18-0}{2}\right)=8$ passengers change queue, i.e. all those who can. The remaining queue for the first bus is made up of $10+\max \left(0, \frac{8+(0-10)}{2}\right)=10$ passengers and that for the second of $0+\min \left(8, \frac{18-0}{2}\right)=0+8=8$ passengers. In other words, in this case equilibrium cannot be achieved. 


\subsection{Queues during the period when both buses load passengers}

For the distribution of passengers after time $a_{m^{\prime}\left(l^{\prime}\right)}$ and before departure of any of the two buses we need to consider the effect of queue length on the newly arriving passengers. In case the queues are of equal length the newly arriving passengers will split also so as to maintain equal queue length if the distribution of passengers allows doing so. That is, we obtain as line (not line set) specific arrival rates $\hat{q}_{m(l), n}(\cdot)$ the same split as in (24). In case the queues are not of equal length, the common line passengers will all join the shorter queue until queue equilibrium is reached. Therefore, in summary, we obtain:

$\hat{q}_{m(l)}(\cdot)=\left\{\begin{array}{cl}\min \left(\max \left(\sum_{\mathcal{L} \in \Omega_{l, \neg l^{\prime}}} q_{\mathcal{L}}, \sum_{\mathcal{L} \in \Omega} q_{\mathcal{L}} / 2\right), \sum_{\mathcal{L} \in \Omega_{l}} q_{\mathcal{L}}\right) & \text { if } \hat{z}_{m(l)}\left(a_{m^{\prime}\left(l^{\prime}\right)}\right)=\hat{z}_{m^{\prime}\left(l^{\prime}\right)}\left(a_{m^{\prime}\left(l^{\prime}\right)}\right) \\ \sum_{\mathcal{L} \in \Omega_{l, \neg l^{\prime}}} q_{\mathcal{L}} & \text { if } \hat{z}_{m(l)}\left(a_{m^{\prime}\left(l^{\prime}\right)}\right)>\hat{z}_{m^{\prime}\left(l^{\prime}\right)}\left(a_{m^{\prime}\left(l^{\prime}\right)}\right) \\ \sum_{\mathcal{L} \in \Omega_{l}} q_{\mathcal{L}} & \text { if } \hat{z}_{m(l)}\left(a_{m^{\prime}\left(l^{\prime}\right)}\right)<\hat{z}_{m^{\prime}\left(l^{\prime}\right)}\left(a_{m^{\prime}\left(l^{\prime}\right)}\right)\end{array}\right.$

Where $\hat{q}_{m(l)}$ will be constant from time $a_{m^{\prime}\left(l^{\prime}\right)}$ until either bus $m(l)$ departs or if equilibrium queues might be reached not at $a_{m^{\prime}\left(l^{\prime}\right)}$ but at a later time. A necessary condition for this is that more passengers join the shorter queue so that one queue is diminishing faster than the other. Equilibrium time, if it exists, can be reached at time interval $\frac{z_{m(l)}\left(a_{m^{\prime}\left(l^{\prime}\right)}\right)-z_{m^{\prime}\left(l^{\prime}\right)}\left(a_{m^{\prime}\left(l^{\prime}\right)}\right)}{\hat{q}_{m^{\prime}\left(l^{\prime}\right)}\left(a_{m^{\prime}\left(l^{\prime}\right)}\right)-\hat{q}_{m(l)}\left(a_{m^{\prime}\left(l^{\prime}\right)}\right)}$ after $a_{m^{\prime}\left(l^{\prime}\right)}$. We can further express the time when either one of the queues has disappeared so that this bus departs as in (26)

$$
\varphi=\min \left(\frac{\hat{z}_{m(l)}\left(a_{m^{\prime}\left(l^{\prime}\right)}\right)}{b_{m}-\hat{q}_{m(l)}\left(a_{m^{\prime}\left(l^{\prime}\right)}\right)}, \frac{\hat{z}_{m^{\prime}\left(l^{\prime}\right)}\left(a_{m^{\prime}\left(l^{\prime}\right)}\right)}{b_{m}-\hat{q}_{m^{\prime}\left(l^{\prime}\right)}\left(a_{m^{\prime}\left(l^{\prime}\right)}\right)}\right)
$$

With this we can express the time interval of diminishing gaps between the two queues as (27)

$$
\tau=\min \left(\frac{\hat{z}_{m(l)}\left(a_{m^{\prime}\left(l^{\prime}\right)}\right)-\hat{z}_{m^{\prime}\left(l^{\prime}\right)}\left(a_{m^{\prime}\left(l^{\prime}\right)}\right)}{\hat{q}_{m^{\prime}\left(l^{\prime}\right)}\left(a_{m^{\prime}\left(l^{\prime}\right)}\right)-\hat{q}_{m(l)}\left(a_{m^{\prime}\left(l^{\prime}\right)}\right)}, \varphi\right)
$$

The resulting dwell time can then be obtained with (28) and overtaking will occur if $w_{m(l)}>w_{m^{\prime}\left(l^{\prime}\right)}$.

$$
w_{m(l)}=\left\{\begin{array}{lc}
a_{m^{\prime}\left(l^{\prime}\right)}-a_{m(l)}+\varphi+\frac{\hat{z}_{m(l), n}\left(a_{m^{\prime}\left(l^{\prime}\right)}+\varphi\right)}{b_{m}-\hat{q}_{m(l), n}\left(a_{m^{\prime}\left(l^{\prime}\right)}+\varphi\right)} & \text { if } \hat{z}_{m(l)}\left(a_{m^{\prime}\left(l^{\prime}\right)}\right)=\hat{z}_{m^{\prime}\left(l^{\prime}\right)}\left(a_{m^{\prime}\left(l^{\prime}\right)}\right) \\
a_{m^{\prime}\left(l^{\prime}\right)}-a_{m(l)}+\tau+\frac{\hat{z}_{m(l), n}\left(a_{m^{\prime}\left(l^{\prime}\right)}+\tau\right)}{b_{m}-\hat{q}_{m(l), n}\left(a_{m^{\prime}\left(l^{\prime}\right)}+\tau\right)} & \text { otherwise }
\end{array}\right.
$$




\subsection{Summary: Queue distribution for front and back buses}

Table 1 summarises the cases that can now be distinguished, assuming, without loss of generality, that bus $m(l)$ is at the stop and then bus $m^{\prime}\left(l^{\prime}\right)$ arrives. Depending on which case applies in (26) the common line passengers will choose the shorter queue or split so that the queues remain equally long. In the third column of the table then the resulting times at which the queues are equally long are denoted, while the fourth column indicates whether overtaking occurs.

In the same way the reversed table can be constructed to obtain the cases if bus $m(l)$ arrives at the bus stop when there is already a bus boarding passengers. We also note that the case that two buses belong to the same line i.e. $l=l^{\prime}$ is also covered in above discussion. In that case all passengers can be considered as common line or "flexible" passengers with choice set $\Omega_{l, l^{\prime}}$ that can board both buses.

Table 1. Effect of initial queues on subsequent queue distribution, equilibrium times and overtaking

\begin{tabular}{|c|c|c|c|c|}
\hline \multirow{2}{*}{$\begin{array}{l}\text { Initial queue at } \\
a_{m^{\prime}\left(l^{\prime}\right)}: \hat{z}_{m(l), n}\end{array}$} & \multicolumn{2}{|c|}{ Queue distribution just after $a_{m^{\prime}\left(l^{\prime}\right)}$} & \multirow{2}{*}{$\begin{array}{l}\text { Resulting time at } \\
\text { which queues are in } \\
\text { equilibrium }\end{array}$} & \multirow{2}{*}{$\begin{array}{l}\text { Does } \quad \text { overtaking } \\
\text { occur? }\end{array}$} \\
\hline & $\hat{q}_{m(l)}$ & $\hat{q}_{m^{\prime}\left(l^{\prime}\right)}$ & & \\
\hline \multirow{3}{*}{$\begin{array}{l}\text { Equilibrium } \\
\text { queues } \\
\sum_{\mathcal{L} \in \Omega} z_{\mathcal{L}}(\cdot) / 2\end{array}$} & $\sum_{\mathcal{L} \in \Omega} q_{\mathcal{L}}(\cdot) / 2$ & $\sum_{\mathcal{L} \in \Omega} q_{\mathcal{L}}(\cdot) / 2$ & $\begin{array}{l}\text { From } a_{m^{\prime}\left(l^{\prime}\right)} \text { until } \\
\text { departure. }\end{array}$ & $\begin{array}{l}\text { No, buses depart at } \\
\text { the same time }\end{array}$ \\
\hline & $\sum_{\mathcal{L} \in \Omega_{l, \neg l^{\prime}}} q_{\mathcal{L}}(\cdot)$ & $\sum_{\mathcal{L} \in \Omega_{l^{\prime}}} q_{\mathcal{L}}(\cdot)$ & \multirow{2}{*}{$\begin{array}{lr}\text { At } \quad a_{m^{\prime}\left(l^{\prime}\right)} & \text { but } \\
\text { queues do not } \\
\text { remain } & \text { in } \\
\text { equilibrium } & \\
\end{array}$} & Yes \\
\hline & $\sum_{\mathcal{L} \in \Omega_{l}} q_{\mathcal{L}}(\cdot)$ & $\sum_{\mathcal{L} \in \Omega_{\neg l, l^{\prime}}} q_{\mathcal{L}}(\cdot)$ & & $\begin{array}{ll}\text { No, bus } & m(l) \\
\text { departs first } & \end{array}$ \\
\hline $\begin{array}{l}\text { Queue of bus } \\
m(l) \text { is longer } \\
\sum_{\mathcal{L} \in \Omega_{l, \neg l^{\prime}}} z_{\mathcal{L}}(\cdot)\end{array}$ & $\sum_{\mathcal{L} \in \Omega_{l, \neg l^{\prime}}} q_{\mathcal{L}}(\cdot)$ & $\sum_{\mathcal{L} \in \Omega_{l^{\prime}}} q_{\mathcal{L}}(\cdot)$ & \multirow[t]{2}{*}{$\begin{array}{l}\text { possibly at } \tau \text { as } \\
\text { obtained from }(27)\end{array}$} & $\begin{array}{l}\text { Not if equilibrium } \\
\text { is reached, } \\
\text { otherwise yes. }\end{array}$ \\
\hline $\begin{array}{l}\text { Queue of bus } \\
m^{\prime}\left(l^{\prime}\right) \text { is longer } \\
\sum_{\mathcal{L} \in \Omega_{l}} z_{\mathcal{L}}(\cdot)\end{array}$ & $\sum_{\mathcal{L} \in \Omega_{l}} q_{\mathcal{L}}(\cdot)$ & $\sum_{\mathcal{L} \in \Omega_{\neg l, l^{\prime}}} q_{\mathcal{L}}(\cdot)$ & & No \\
\hline
\end{tabular}

We note that this table omits some possibly even more complex cases: Let bus $m(l)$ arrive first followed by a bus of a different line $l^{\prime}$ that is arriving while the bus of line $l$ is still boarding. In case there are few common line passengers and the arrival rate of passengers for bus $l$ is high, it might mean that the first bus is hence overtaken. It might now be that another bus is arriving while bus $m(l)$ is still boarding passengers. This correction in dwell time due to a third bus interacting with bus $m(l)$ and relieving its load is not taken into consideration in above algorithm. The error will be usually small though unless, possibly, if all three buses are from different lines and if there are high rates of passenger flows who have attractive line sets consisting of two of the three lines. In that case, 
denoting the lines in order of bus arrival, bus 2 might not have relieved the load of bus 1 significantly if there are many passengers with choice set $\{1,3\}$. If there are few passengers with choice set $\{2\}$, bus 2 will leave early and the split of queues when bus 3 arrives would need additional consideration. Algorithm 2 could be amended accordingly and in line with our discussion above. In the following case study we limit ourselves though to the cases of two lines.

\subsection{Bus trajectories with overtaking}

Combining this discussion with the state equations shown in Algorithm 1, an alternative Algorithm 2 can be obtained.

Algorithm 2: Bus trajectories with overtaking

Initialisation as in Algorithm 1

For each stop $n$ in increasing order

Sort buses according to arrival time at stop

For each bus $m \in \mathcal{L}$ in order of increasing arrival times

If $a_{m(l), n}>d_{m^{\prime}\left(l^{\prime}\right), n} \forall m^{\prime} \mid a_{m(l), n}>a_{m^{\prime}\left(l^{\prime}\right), n}:$ Bus $m$ is front bus at the stop

Obtain $w_{m(l) n}$ as in Eq. (16) and $d_{m(l) n}$ as in (19)

Test if $d_{m(l), n}>a_{m^{\prime}\left(l^{\prime}\right), n} \forall m^{\prime} \mid a_{m(l), n}<a_{m^{\prime}\left(l^{\prime}\right), n}$

If yes, revise dwell time according to cases in Table 1 and (28)

Else: Bus is back bus of two at bus stop

Obtain dwell time according to (28)

Obtain $d_{m(l) n}$ with (19) and $a_{m(l), n+1}$ with (18).

\subsection{Alternative formulations}

In addition to the overtaking case introduced above and the non-overtaking case, one might also define an "intermediate case" where buses are allowed to overtake buses of different lines but not of the same line. This case appears to be the operating practice in many countries, including in Japan, out of "fairness" considerations. That is, the FIFO principle should not be violated in that passengers who queued and boarded earlier should also have the right to arrive earlier compared to passengers who 
boarded the same line later. In the algorithms this means hence that the departure time will be obtained instead by

$$
d_{m(l) n}=\max \left\{d_{m(l)-1, n}+\varepsilon, a_{m(l), n}+w_{m(l), n}\right\}
$$

\section{Evaluation measures}

In order to quantify the benefits of overtaking and common line stops evaluation measures are needed. Firstly, the total passenger waiting time for a single stop and line can be obtained by

$$
\widetilde{w}_{l n}=\sum_{m(l)} \int_{\Delta_{\mathcal{L}, m(l), n}} t q_{l n}(t) d t
$$

As we assume constant arrival rates (30) is likely to lead to an overestimation of the true waiting time though if we assume a constant number of buses and delays. The bunching effect means that the last bus is likely to arrive later at the last bus stop. This in turn means that more passengers have arrived which hence means an unequal comparison in terms of number of passengers. To account for this, we consider the average waiting time of each passenger for each line to be a better index to assess the performance of the service from the view of users, which can be expressed by

$$
\bar{w}_{l}=\frac{\sum_{n} \sum_{m(l)} \int_{\Delta_{\mathcal{L}, m(l), n}} t q_{l n}(t)}{\sum_{n} \sum_{m(l)} \int_{\Delta_{\mathcal{L}, m(l), n}} q_{l n}(t)}
$$

Secondly, we utilise the standard deviation of $\Delta_{m, n}$ with respect to each line in (32) as an indicator of service regularity. We define this measure line specific as we are particularly interested in understanding the knock-on effect of delays on one line to regularity of an initially unaffected line.

$\sigma_{l}=\sqrt{\frac{\sum_{n} \sum_{m(l)}\left(\Delta_{\mathcal{L}, m(l) n}-\bar{\Delta}_{\mathcal{L}, m(l) n}\right)^{2}}{M(l) N(l)}}$

In addition, the maximum waiting times might also be of concern as some long waiting times might be perceived worse than a number of slightly longer than usual waiting times. We specifically pay attention to the maximum waiting time at the last stop $N(l)$ as in (34) where the service is generally worst. If the maximum waiting time of the unreliable line at the last stop is reduced due to common lines, it indicates that the more reliable line has helped the less reliable line to recover service regularity over the common line section.

$\widehat{W}^{N}{ }_{l}=\max _{m(l)} \Delta_{\mathcal{L}, m(l) N(l)}$ 


\section{Case study}

\subsection{Specifications}

We consider the simple two line scenario illustrated in Figure 1 with 10 stops. The red line is referred to as $L 1$ and the blue one as $L 2$ hereafter. The two bus lines both run with the same frequency of $h=6 \mathrm{~min}$ and are scheduled to arrive at stops with a constant inter-arrival time so that every $3 \mathrm{~min}$ a bus serves the stop. We choose these simple settings as we our main conclusions are best illustrated by these. Choosing different headways for the lines and/or varying inter-arrival times is though not a model restriction. Our third parameter is the arrival-to-loading ratio where we assume $k=0.25$ for all stops. We assume that an initial random delay of $2 \mathrm{~min}$ occurs for the $2^{\text {nd }}$ bus of Line 1 at stop 2 : $\rho_{2(1) 2}=2$. This means that the first buses of both lines are unaffected and hence run with the expected headways and encounter the same (expected) dwell times at the stop.

To evaluate the effect of common lines we test different common-line stop specifications. Firstly, we test the effect of Stops 2 to 8 being common-line stops or not. Stops 9 and 10 are never common-line stops in order to illustrate the effect that buses might split again after the section of route they serve together. In case none of the bus stops is set as common-line stop, clearly $L 2$ is unaffected by the delay that occurred to $L 1$. Generally we expect, the more common lines, the more $L 1$ can recover, but the more the operation of $L 2$ will be disturbed.

At each stop we thus have an arrival rate vector $\left(q_{\{1\} n}, q_{\{2\} n}, q_{\{1,2\} n}\right)$ where the first two elements denote the fixed demand for $L 1$ and $L 2$ and the third element describes the flexible demand of common-line users to whom both $L 1$ and $L 2$ are attractive. We vary the distribution of these arrival rates in Section 7.2. In Section 7.3 we then assess the service performance under all possible combinations of common-line stops within this corridor.

\subsection{Delays and trajectories in case all stops are common line stop}

We first focus on a scenario where all stops from 2 to 8 are common-line stops. We test three different percentages of common-line users, distinguishing the cases with or without overtaking. The three demand levels are for $q_{\{1,2\} n}$ equals to $0 \%, 20 \%$ and $80 \%$ of total boarding demand respectively. This leads to six scenarios; the resulting bus trajectories are shown in Figure 3.

Firstly, we observe that expected waiting times of passengers for both lines are reduced with the increase of common-line users as these can take whichever bus arrives first. Comparing the trajectories and indices shown in the left column (without overtaking) to those in the right column (with overtaking) by each row, it is obvious that common lines are less effective in case overtaking is 
not possible (or not allowed). The initial delay to the red bus causes it to be further delayed downstream and the green line cannot give enough support to the red line as overtaking is not possible. In case of higher common-line user percentage, worsening service irregularity for both lines is the consequence. Therefore we observe that common line stops are preferably implemented in systems where overtaking is possible. If not, only the negative effect of bunching (spill-over of delay to other lines) occur but the positive effects (service recovery on initial delayed line) cannot be utilized. However, in case overtaking is allowed, the common lines appear to improve the service if there are sufficient common line users. This is rational because whichever bus finishes boarding first can leave and arrive at the downstream stops sooner. In accordance with this, we observe that the standard deviation of departure intervals for the initially delayed line in general reduces if the percentage of common line users increases. The flip-side of this, that is increases in service regularity for the second line, appear to be smaller compared to the benefits for line 1. We illustrate this non-linear relationship further in Figure 4. Interestingly the service appears to be least reliable if only roughly half of all passengers are common line passengers. 


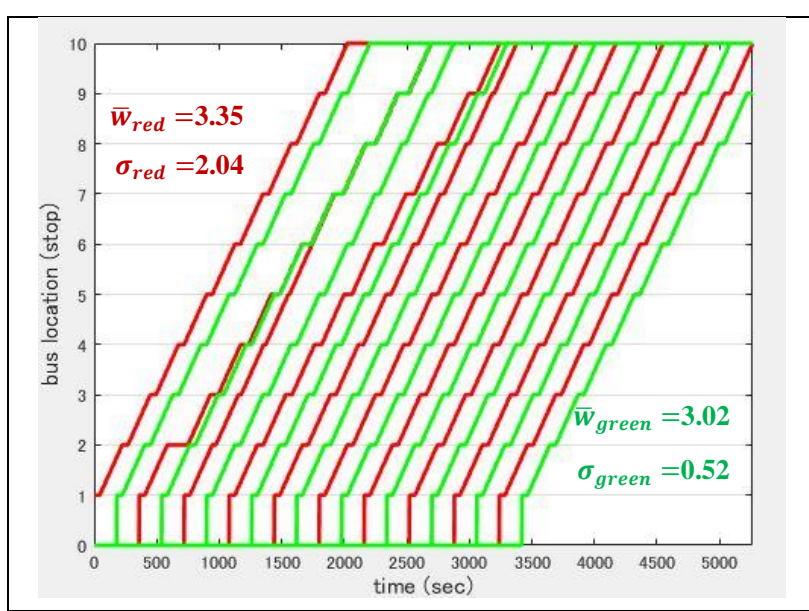

(a) no common-line user, no overtaking

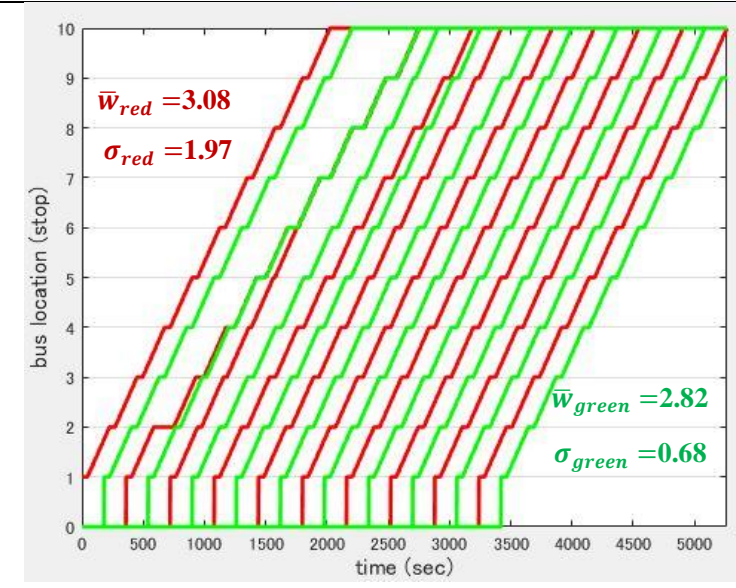

(c) $20 \%$ is common-line user, no overtaking

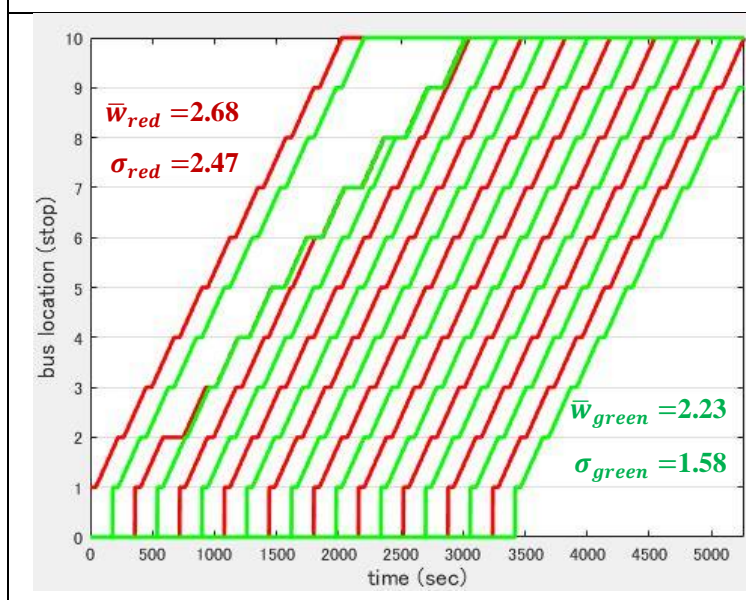

(e) $80 \%$ is common-line user, no overtaking

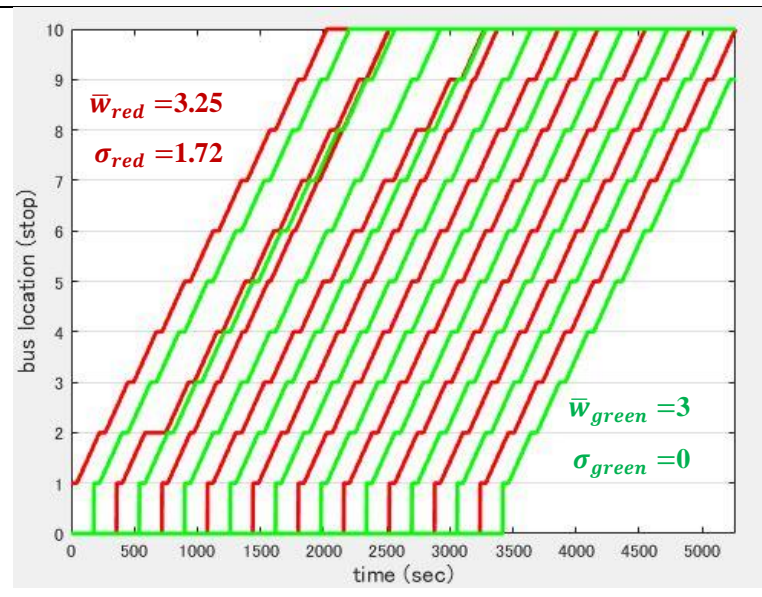

(b) no common-line user, overtaking

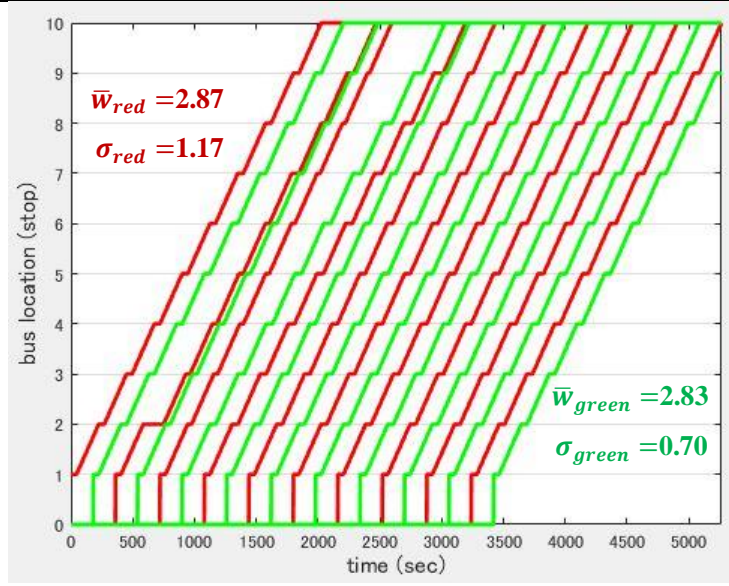

(d) $20 \%$ is common-line user, overtaking

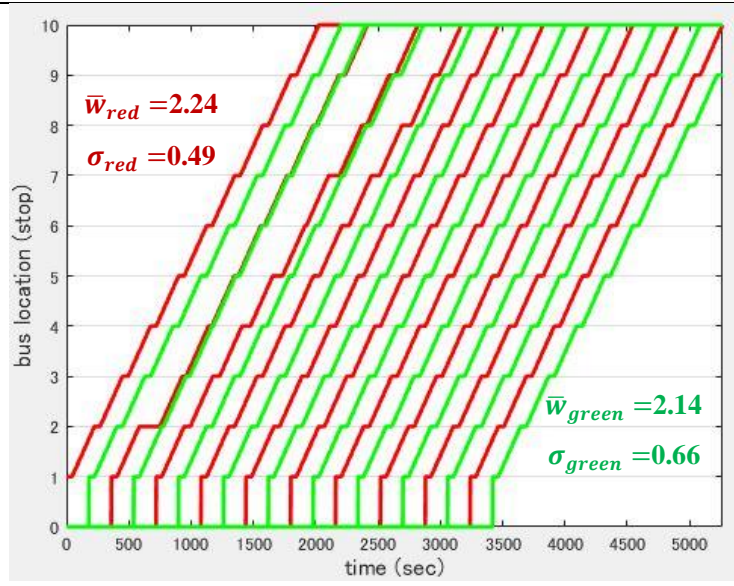

(f) $80 \%$ is common-line user, overtaking

Figure 3 Bus trajectories under different common-line user percentages and distinguished by whether overtaking is allowed or not; indices have the unit [min] 


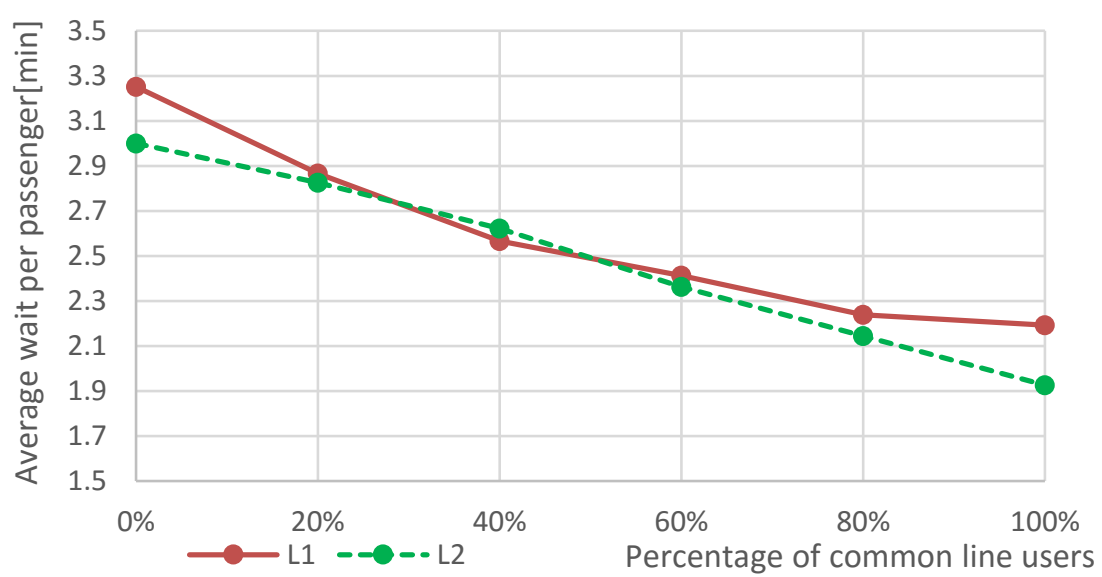

(a) Average waiting time per passenger

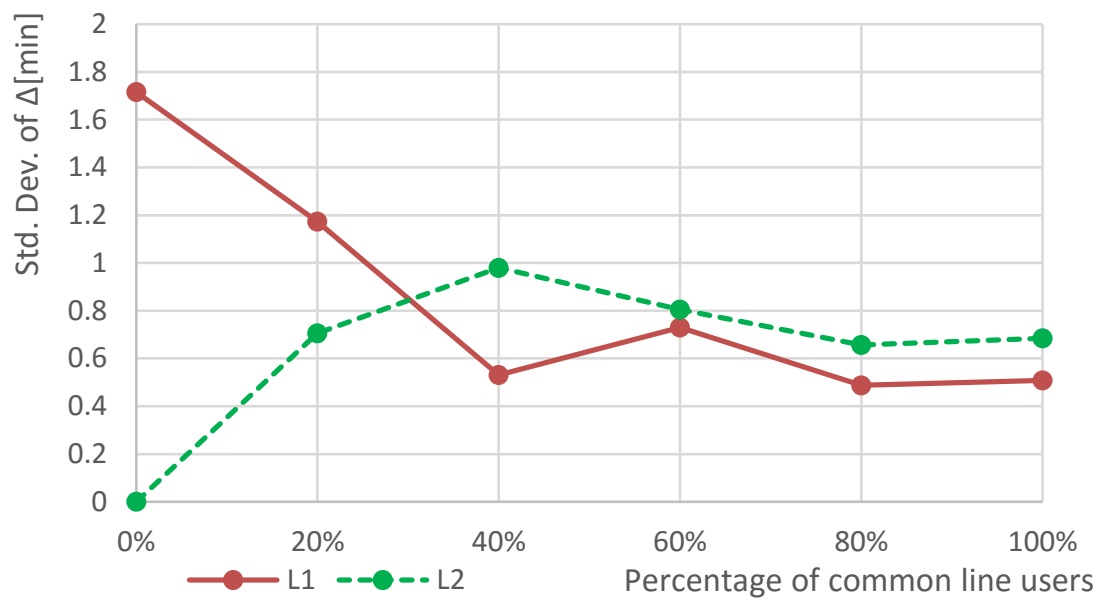

(b) Std. Dev. of $\Delta$

Figure 4 Service performance comparison with different percentage of common line users (with overtaking)

\subsection{Tests with different combinations of common-line stop designs}

Whereas in the previous section we varied the common line demand in this section we focus on bus stop design and test for the effect of combinations of common line and non-common-line stops. Since each stop from 2 to 8 can have two layouts, in total we have $2^{7}=128$ scenarios. We assume that $80 \%$ of users are common-line users.

Figure 5 first shows an evaluation of these 128 scenarios distinguishing the case without and with overtaking. The average waiting time per passenger for the two different lines is used evaluation criteria. The scenarios are grouped (and colour-coded in Fig. 5) by the number of common-line stops the scenario has, regardless where they are located. There are eight groups with respectively 0,1 , 
$2, \ldots 7$ common line stops. A general trend emerges in Fig. 5 that the more common line stops the network has, the less the average waiting time. A few scenarios with three common line stops have higher average wait time than some of the scenarios with four common line stops, suggesting that a strategic implementation of three common line stops can lead to as much delay reductions as four common line stops.

We also observe that the average wait times for Line 1 are always greater than those for Line 2 in Figure 5a (without overtaking) and in general equal greater in Figure 5b (with overtaking). This can be expected as we assume that the initial delay occurs on Line 1 and that the demand distribution for both lines is identical. Therefore Line 2 can absorb some delay of Line 1 but will still perform better than the line that was affected initially.

Comparing Figures $5 \mathrm{a}$ and $\mathrm{b}$ we find that with larger number of common line stops, the average passenger waiting time for Line 1 can be reduced by as much as $20 \%$ when overtaking at bus stops is allowed. This suggests that allowing for overtaking is particularly useful if there are long stretches of common line stops.

In Figure 6 we repeat an illustration of the case shown in Figure 5b and in addition vary the headway of Line 2 (all other settings remain the same). Whereas in our previous tests both lines had a headway of 6 minutes in this case we also illustrate the case of Line 2 having a headway of 8 and 20 minutes. This means that clearly the average waiting time of passengers boarding Line 2 increases. If there are no common lines the average wait for Line 2 passengers equals half the headway. If there are many common line stops and Line 2 has a low frequency, a trade-off relationship can be seen. One the one hand, the assumption of $80 \%$ common line passengers means that many of the passengers who board Line 2 did not wait long as they only happen to board Line 2 if it arrives before Line 1 . One the other hand, the longer the headway for Line 2, the more passengers will board Line 1 and hence increase dwell time and reduce Line 1 service regularity. Therefore, as shown for the case of Line 2 having a long headway of 20min, in fact the average waiting time of passengers boarding Line 1 increases. Overall though, the benefits in total waiting time reduction for all passengers are obvious considering the different scale of the two axes. 


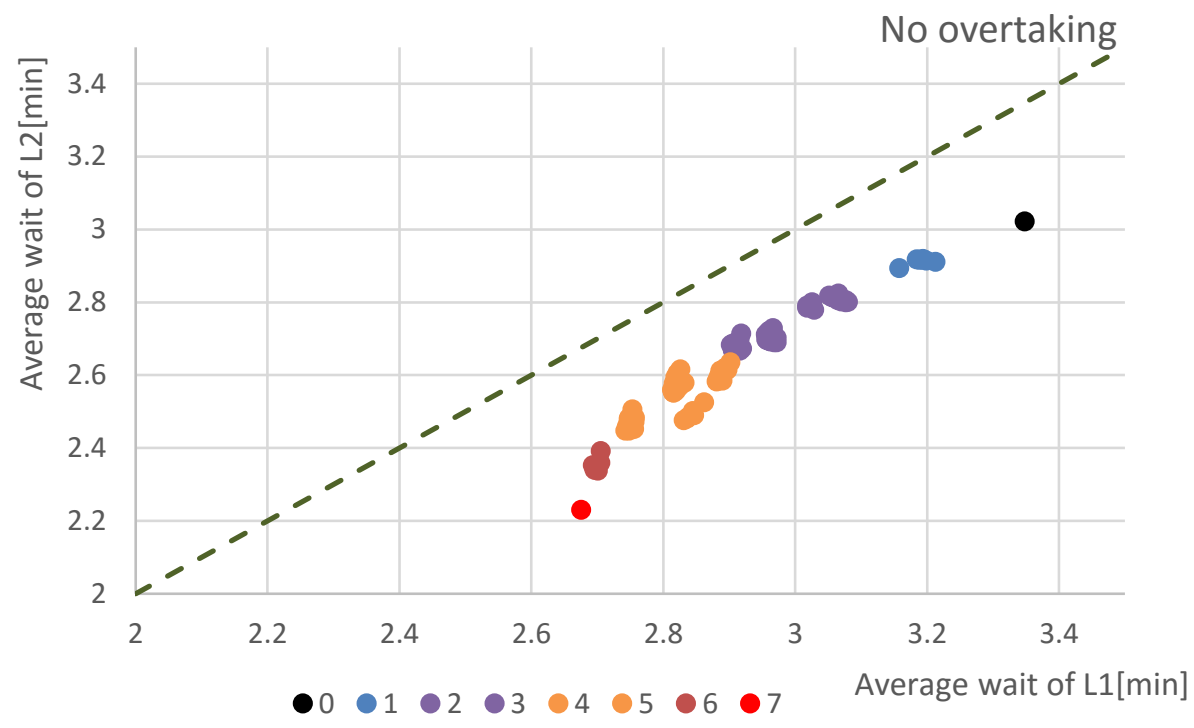

(a) without overtaking

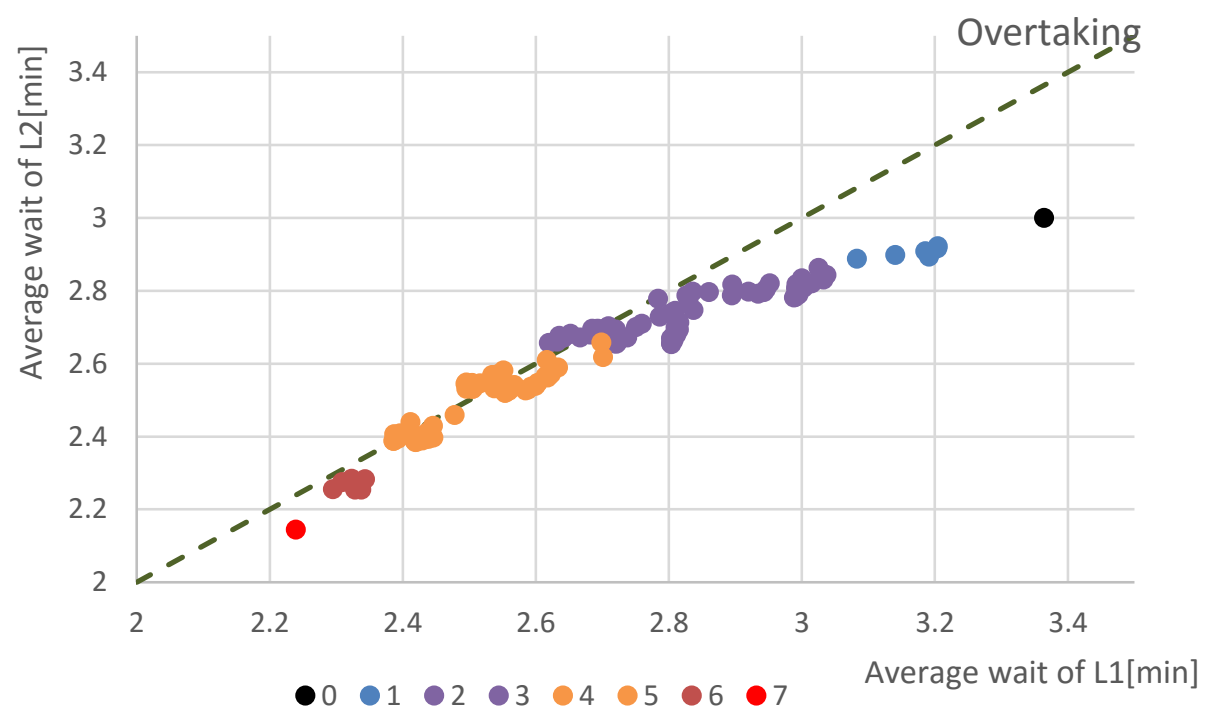

(b) with overtaking

Figure 5 Average waiting time for different number of common-line stops. 


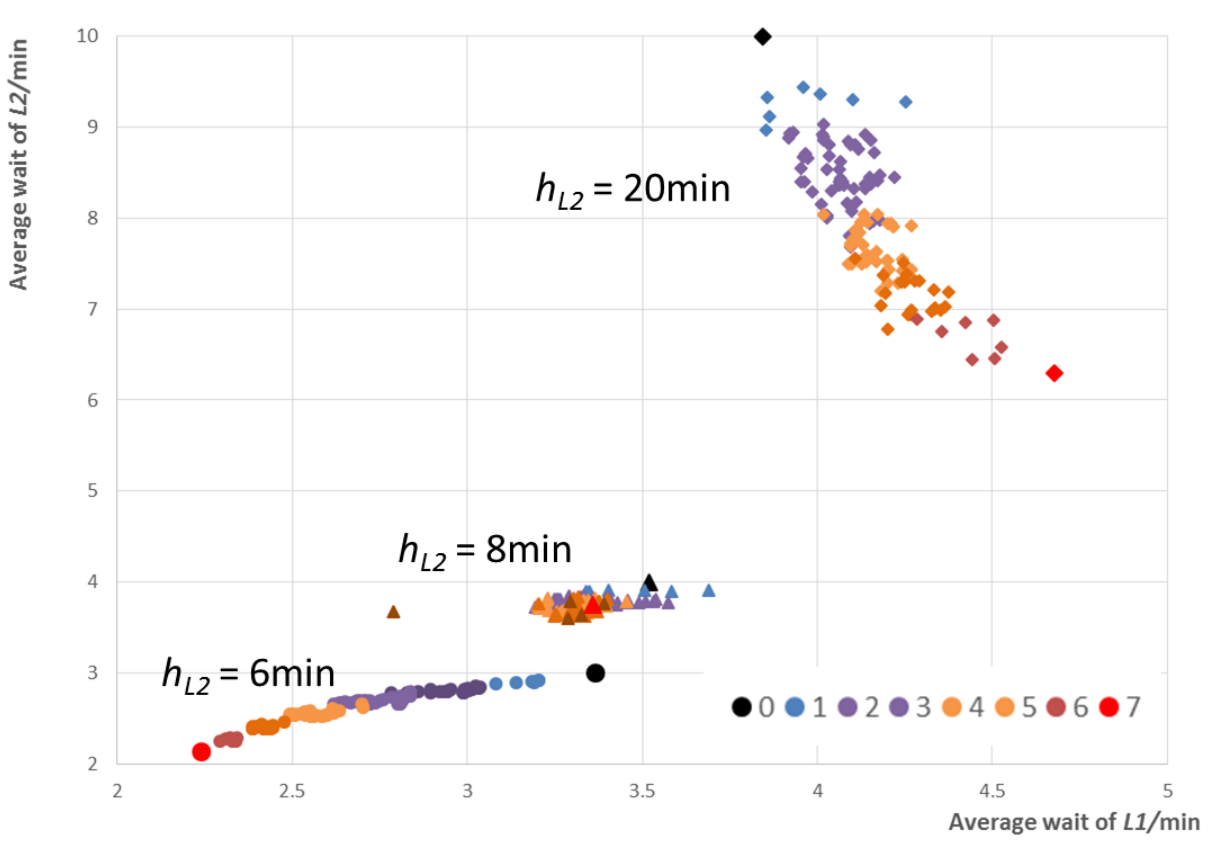

Figure 6 Average waiting time for different Line 2 headways and different number of common line stops

We return to the case of equal line headways $\left(h_{L 1}=h_{L 2}=6 \mathrm{~min}\right)$. Figure 7 shows the standard deviation of wait time for all stops. The figure illustrates the advantages to maintain service regularity in case common lines are introduced. If all stops are converted into common-line stops, the standard deviation of $\Delta$ reaches the minimum for Line 1 . Generally solutions with six or seven stops are on or near the Pareto optimal front whereas reducing the number of common line stops can lead to solutions where one might argue that the reduction in service irregularity for Line 1 is not offset by the irregularity reductions for Line 2 .

Finally, as a further evaluation index, Figure 8 plots the maximum $\Delta$ for Stop 9 at the end of the possible common line section when the services split again. Clearly if there are no common lines the second line is not affected by the initial delay to Line 1 so that $\Delta$ is equal to the service headway. The presence of common line stops reduces the maximum delay to Line 1 significantly and can be achieved by only increasing the maximum delay on Line 2 slightly. Further interesting to note is the generally non-symmetric pattern created by our 128 scenarios. 


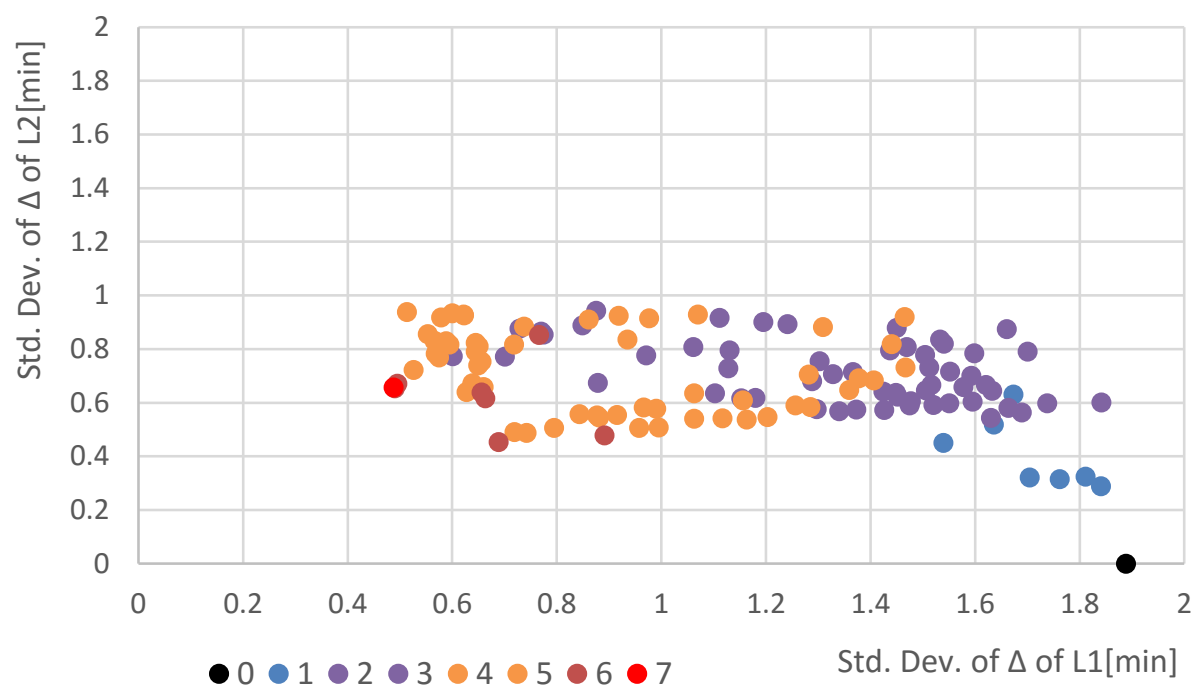

Figure 7 Standard deviation of $\Delta$ (with overtaking)

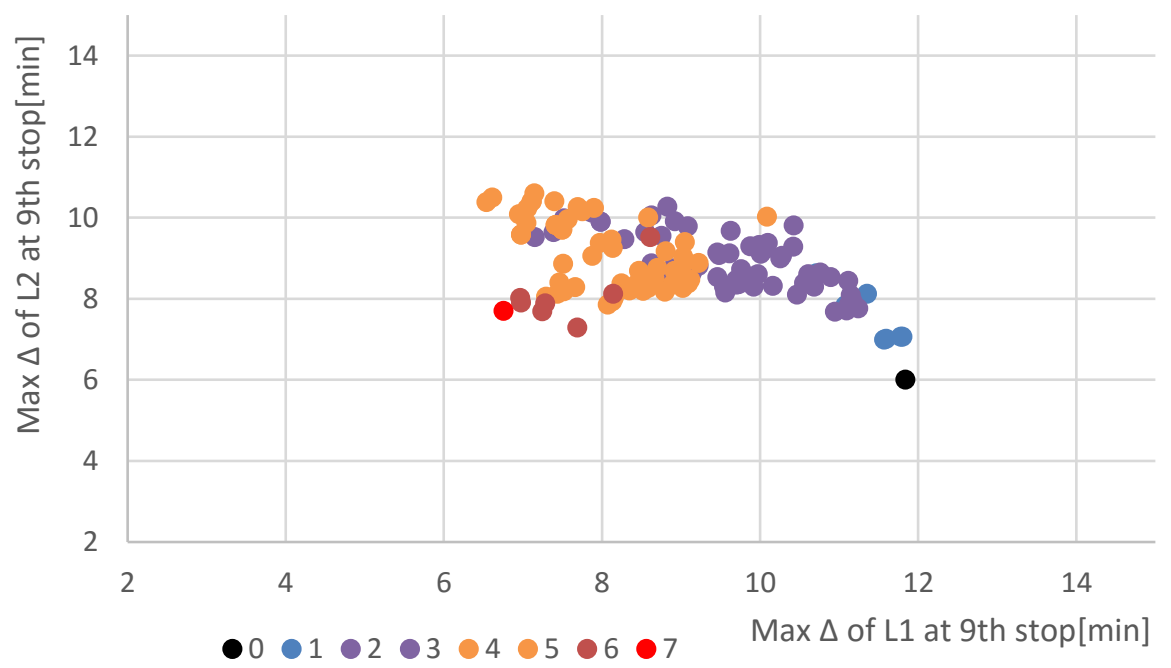

Figure 8 Max $\Delta$ at the end of common section (with overtaking)

\section{Conclusions and further work}

The literature on bus bunching has focused mainly on various forms of holding strategies to reduce the bus bunching effect. However, every holding of a bus is an additional delay whereas we propose here that network layout might be utilised to support service regularity. This paper instead discusses the effect of common lines on bus bunching. We formulated state equations to obtain bus trajectories to obtain theoretical insights. We further envisage this approach to be useful for transit network 
planning where one wants to test a large number of network and bus stop configurations. In the model formulation we introduced in particular the possibility of overtaking among the buses as we believe this was not explicitly formulated in previous literature (except for simulation approaches). Furthermore, a main contribution has been the formulation of a queuing model that considers explicitly the behaviour of passengers according to their choice set. We assume that passengers want to minimise their waiting time and hence form queues of equal length which tends to favour bunching. Our case study illustrates that the presence of common lines can significantly reduce service irregularity. Common lines will have a positive effect on service irregularity specifically if overtaking is allowed and possible. If an operator can only transform some stops into common line stops we find that still significant benefits can be obtained though the negative effects of delay spreading to more reliable lines can be considerable compared to the gain in service regularity for lines prone to delays.

Our scenario tests all assumed that there are no delays except for one initial delay to one of the bus lines and the resulting effects. We did so in order to clearly illustrate the secondary bunching effects. Modelling more general cases of various random delays occurring to buses between stops (traffic lights, congestion etc) or at stops (e.g. passengers requiring additional time for cash handling) does though pose no theoretical challenges as one could generate random $\boldsymbol{\rho}_{l}$ matrices for delays at stops as well as between stops. The main reason for distinguishing two models in Sections 4 and 5 are due to different passenger behaviour: The travel time minimising passenger will have no incentives to board a bus stuck behind another one, whereas, if buses depart whenever passengers on-board, queues of equal length will form.

A number of other further research issues appear important. To increase the realism of the case study we highlight the following issues already noted in various sections of this paper: The role of timedependent loading factors, considering passenger groups with different common line sets, consideration of alighting times as well as bus capacity constraints. In general we would expect that considering these factors, in particular capacity problems, would amplify the propagation of delays, as crowded buses tend to require more time per passenger to complete boarding and alighting. Consideration of crowding would further possibly lead to a revised queueing model in which passengers might predict that crowded buses require more dwell time at subsequent stops and hence prefer to board less congested buses as they tend to complete alighting at stops faster. 


\section{Acknowledgements}

The first two authors acknowledge various comments received on early versions of this work by colleagues at Kyoto University as well as funding received from JSPS Grant "Kiban B" 26289174. The fourth author (R.Liu) acknowledges the financial support from UK Rail Safety and Standard Board under Project RSSB-T1071. 


\section{Appendix: Delay of the first bus and subsequent buses}

Proposition: Increasing the sequence of common line stops reduces the maximum delay of the first delayed bus for constant $k_{l n}$.

Proof: Assume there is no common line stop, and an initial random delay $\rho_{m(l) i}$ at stop $i$. Let $d_{m(l) i}^{S}$ be the scheduled departure of bus $m(l)$ at stop $i$. Then the delay at stop $i$ is $\delta_{m(l) i}=d_{m(l) i}-d_{m(l) i}^{S}=$ $\rho_{m(l) i}$. At the following stop the bus is delayed further due to $\Delta_{\mathcal{L}, m(l) i+1}>\Delta^{S}{ }_{\mathcal{L}, m(l) i+1}=h_{l}$, where $h_{l}$ denotes the scheduled headway of the line. Since we assume that no further delays occur between stops $\Delta_{\mathcal{L}, m(l) i+1}-h_{l}=\rho_{m(l) i}$ and the delay at stops can be derived as:

$$
\delta_{m(l) i+1}=\rho_{m(l) i}+\rho_{m(l) i} k_{i+1, l}
$$

Assuming that $k$ is constant across stops and buses this hence leads to

$$
\delta_{m(l) i+2}=\delta_{m(l) i+1}+\delta_{m(l) i+1} k=\rho_{m(l) i}+2 \rho_{m(l) i} k+\rho_{m(l) i} k^{2}
$$

Or in general for downstream stops from $i$

$$
\delta_{m(l), i+n}=\delta_{m(l), i+n-1}+\delta_{m(l), i+n-1} k=\rho_{m(l) i}(1+k)^{n}
$$

Utilising that the stop departure from a stop $j$ can be derived from arrival time $a_{m(l) j}$ plus dwell $\Delta_{\mathcal{L}, m(l) j} k$ the departure time considering delays can hence also be obtained accordingly

$$
d_{m(l) j}=d_{m(l) j}^{S}+\delta_{m(l) j}=a_{m(l) j}^{S}+\delta_{m(l), j-1}+\Delta_{\mathcal{L}, m(l) j} k
$$

Above assumed that none of the stops is a common lines stop. Assume now that stop $j{ }^{\prime}>i$ is the first common line stop after stop $i$. Comparing this to the case $j$ where the stop is not a common lines stop leads to $\Delta_{\mathcal{L}^{\prime}, m(l) j^{\prime}} \leq \Delta_{\mathcal{L}, m(l) j}$ since in the case of $j^{\prime}$ set $\mathcal{L}^{\prime}$ includes line $l$ plus at least another line and it follows that $d_{m(l) j^{\prime}} \leq d_{m(l) j}$. Clearly any further common line stops downstream of $j$ will further reduce the delay. Q.E.D.

The proof above only holds for the first bus of the line. The second bus of line $l$ at stop $j$ can be further delayed if $j$ is a common line stop due to the earlier departure of the first bus. It can, however, also be less delayed if a bus of another line was able to pick most of the passengers by the time the second bus arrives. 


\section{References}

Bartholdi, J.J., Eisenstein, D.D. (2012). A self-coordinating bus route to resist bus bunching. Transportation Research, Part B, 46, 481-491.

Bowman and Turnquist (1981) Service frequency, schedule reliability and passenger wait times at transit stops. Transportation Research, Part A, 15(6), 465-471.

Boyd, C.W. (1987) Notes on the theoretical dynamics of intermittent public passenger transportation systems. Transportation Research, Part A, 17(5), 347-354.

Cats, O., Nabavi, A., Larijani, Burghout, W. and Koutsopoulos, H.N. (2012) Impacts of holding control strategies on transit performance: A bus simulation model analysis. Transportation Research Record, 2584, 51-58.

Chapman, R.A. and Michel, J.F. (1978) Modelling the tendency of buses to form pairs. Transportation Science, 12(2), 165-175/

Daganzo, C. F. (2009) A headway-based approach to eliminate bus bunching: Systematic analysis and comparisons. Transportation Research, Part B, 43(10), 913-921.

Daganzo, C.F., Pilachowski, J. (2011). Reducing bunching with bus-to-bus cooperation. Transportation Research, Part B, 45, 267-277.

Eberlein, X.J., Wilson, N.H.M., Bernstein, D. (2001) The holding problem with real-time information available. Transp. Sci., 35(1), 1-18.

Fonzone, A., Schmöcker, J.-D. and Liu, R. (2015). A model of bus bunching under reliability-based passenger arrival patterns. Selected Proceedings of the $21^{\text {th }}$ International Symposium on Transportation and Traffic Theory (ISTTT). Transportation Research Part C, 59, 164-182.

Hernández, D., Muñoz, J.C., Giesen, R. and Delgado, F. (2015) Analysis of real-time control strategies in a corridor with multiple bus services. Transportation Research, Part B, 78, 83-105.

Hickman, M.D. (2001) An analytic stochastic model for the transit vehicle holding problem. Transportation Science, 35(3), 215-237.

Hollander, Y. and Liu, R (2008) Estimation of the distribution of travel times by repeated simulation. Transportation Research, Part C, 16, 212-231.

Jolliffe, J.K and Hutchinson, T.P. (2001) A behavioural explanation of the association between bus and passenger arrivals at a bus stop. Transportation Science. 9(3), 248-281.

Liu, R. and Sinha, S. (2007) Modelling urban bus service and passenger reliability. Proceedings of the International Symposium on Transportation Network Reliability, The Hague, July 2007.

Nagatani, T. (2001) Interaction between buses and passengers on a bus route. Physica A, 296, 320330.

Newell, G.F. (1974) Control of pairing of vehicles on a public transportation route, two vehicles, one control point. Transportation Science, 8(3), 248-264. 
Newell, G.F. and Potts, R.B. (1964). Maintaining a bus schedule. Proceedings $2^{\text {nd }}$ Australian Road Research Board, 2, 388-393.

Osuna, E.E. Newell, G.F. (1972) Control strategies for an idealized bus system. Trans. Sci. 6(1), 5271.

Pilachowski, J.M. (2009) An Approach to Reducing Bus Bunching. PhD Thesis, University of California, Berkeley.

Potts, R.B. and Tamlin, E.A. (1964) Paring of buses. Proceedings of $2^{\text {nd }}$ Australian Road Research Board (ARRB) Conference, 2(2), 3-9.

Sorratini, J., Liu, R. and Sinhan, S. (2008) Assessing bus transport reliability using microsimulation. Transport Planning \& Technology, 31(3), 303-324.

Sun, W. and Schmöcker, J.-D. (2016). Considering Passenger Choices and Overtaking in the Bus Bunching Problem. Presented at the $95^{\text {th }}$ Annual Meeting of the Transportation Research Board. Washington D.C., U.S.

Sun, L., Tirachini, A., Axhausen, K.W., Erath, A. and Lee, D.-H. (2014) Models of bus boarding and alighting dynamics. Transportation Research Part A, 69, 447-460. 